MATHEMATICS OF COMPUTATION

Volume 75, Number 256, October 2006, Pages 1997-2019

S $0025-5718(06) 01843-6$

Article electronically published on June 16, 2006

\title{
THE MONIC INTEGER TRANSFINITE DIAMETER
}

\author{
K. G. HARE AND C. J. SMYTH
}

\begin{abstract}
We study the problem of finding nonconstant monic integer polynomials, normalized by their degree, with small supremum on an interval $I$. The monic integer transfinite diameter $t_{\mathrm{M}}(I)$ is defined as the infimum of all such supremums. We show that if $I$ has length 1 , then $t_{\mathrm{M}}(I)=\frac{1}{2}$.

We make three general conjectures relating to the value of $t_{\mathrm{M}}(I)$ for intervals $I$ of length less than 4 . We also conjecture a value for $t_{\mathrm{M}}([0, b])$ where $0<b \leq 1$. We give some partial results, as well as computational evidence, to support these conjectures.

We define functions $L_{-}(t)$ and $L_{+}(t)$, which measure properties of the lengths of intervals $I$ with $t_{\mathrm{M}}(I)$ on either side of $t$. Upper and lower bounds are given for these functions.

We also consider the problem of determining $t_{\mathrm{M}}(I)$ when $I$ is a Farey interval. We prove that a conjecture of Borwein, Pinner and Pritsker concerning this value is true for an infinite family of Farey intervals.
\end{abstract}

\section{INTRODUCTION AND RESULTS}

In this paper we continue a study, recently initiated by Borwein, Pinner and Pritsker [2], of the monic integer transfinite diameter of a real interval. We write the normalized supremum on an interval $I$ as

$$
\|P\|_{I}^{*}:=\sup _{x \in I}|P(x)|^{1 / \operatorname{deg} P} .
$$

Note that this is not a norm. Then the monic integer transfinite diameter $t_{\mathrm{M}}(I)$ is defined as

$$
t_{\mathrm{M}}(I):=\inf _{P}\|P\|_{I}^{*},
$$

where the infimum is taken over all nonconstant monic polynomials with integer coefficients. We call $t_{\mathrm{M}}(I)$ the monic integer transfinite diameter of $I$ (also called the monic integer Chebyshev constant [1, 2]). Clearly $t_{\mathrm{M}}(I) \geq t_{\mathbb{Z}}(I)$, where $t_{\mathbb{Z}}(I)$ denotes the integer transfinite diameter, defined using the same infimum, but taken over the larger set of all nonconstant polynomials with integer coefficients [3, 4, 5]. Further $t_{\mathbb{Z}}(I) \geq \operatorname{cap}(I)$, the capacity or transfinite diameter of $I$ [6, 14, which can be defined again using the same infimum, but this time taken over all nonconstant monic polynomials with real coefficients. It is well known that $\operatorname{cap}(I)=|I| / 4$ for an interval $I$ of length $|I|$. Further, if $|I| \geq 4$, then $t_{\mathbb{Z}}(I)=t_{\mathrm{M}}(I)=\operatorname{cap}(I)$ by 2], so that the challenge for evaluating $t_{\mathrm{M}}(I)$, as for $t_{\mathbb{Z}}(I)$, lies in intervals with

Received by the editor April 21, 2005 and, in revised form, June 20, 2005.

2000 Mathematics Subject Classification. Primary 11C08; Secondary 30C10.

Key words and phrases. Chebyshev polynomials, monic integer transfinite diameter.

Research of the first author was supported in part by NSERC of Canada and a Seggie Brown Fellowship, University of Edinburgh. 
$|I|<4$. For these intervals we know from [2, Prop. 1.2] that $t_{\mathrm{M}}(I)<1$. However, in contrast to the study of $t_{\mathbb{Z}}(I)$, it is possible to evaluate the monic integer transfinite diameter $t_{\mathrm{M}}(I)$ exactly over some such intervals.

Our first result is the following.

Theorem 1.1. All intervals $I$ of length 1 have $t_{\mathrm{M}}(I)=\frac{1}{2}$. In fact, slightly more is true: if $1 \leq|I| \leq 1.008848$, then $t_{\mathrm{M}}(I)=\frac{1}{2}$.

Furthermore for any $b<1$ there is an interval $I$ with $|I|=b$ and $t_{\mathrm{M}}(I)<\frac{1}{2}$, while for $b>1.064961507$ there is an interval $I$ with $|I|=b$ and $t_{\mathrm{M}}(I)>\frac{1}{2}$.

The proof, which is essentially a corollary of Theorem 1.2 (a) below, is discussed in Section 5 .

The numbers 1.008848 and 1.064961507 in Theorem 1.1, like most numerical values given in this paper, are approximations to some specific algebraic number. These numbers are rounded in the correct direction, if necessary, to ensure an inequality still holds. The polynomial equations that they satisfy are given within the text. We have tried to do this for all numerical values.

To measure the range of lengths of intervals having a particular monic integer transfinite diameter $t$, we introduce the following two functions:

$$
\begin{aligned}
& L_{-}(t):=\inf _{I}\left\{|I|: t_{\mathrm{M}}(I)>t\right\}, \\
& L_{+}(t):=\sup _{I}\left\{|I|: t_{\mathrm{M}}(I) \leq t\right\} .
\end{aligned}
$$

It follows from [2, Prop. 1.3] that both $L_{-}(t)$ and $L_{+}(t)$ are nondecreasing functions of $t$. Also $L_{-}(t) \leq L_{+}(t)$; see Lemma 3.1(a) below. We give (Proposition 3.1) a general method for finding upper and lower bounds for $L_{-}(t)$ and $L_{+}(t)$, and apply these methods to get such bounds for $\frac{1}{2} \leq t \leq 1$. They are constructive, using both the LLL basis-reduction algorithm and the Simplex method. These techniques were first applied in this area by Borwein and Erdélyi [3], and then by Habsieger and Salvy [7. These bounds are given in Theorem 4.1] and Proposition 4.1; see also Figures 11 and 2 ,

At $t=\frac{1}{2}$, we pushed this method further and were able to say more.

Theorem 1.2. We have

(a) $1.008848 \leq L_{-}\left(\frac{1}{2}\right) \leq 1.064961507$ and

(b) $\sqrt{2} \approx 1.41421 \leq L_{+}\left(\frac{1}{2}\right) \leq 1.4715$.

Further properties of $L_{+}$and $L_{-}$are given in Lemma 3.1 ,

\section{Definitions, COnjeCtures, AND FURTher Results}

In this section, we state some old and some more new results, and (perhaps a little recklessly) make four conjectures.

The following result is simple but fundamental. It is useful for determining lower bounds for $t_{\mathrm{M}}(I)$.

Lemma BPP (Borwein, Pinner, and Pritsker [2, p. 1905]). Let $Q(x)=a_{d} x^{d}+$ $\cdots+a_{0}$ be a nonmonic irreducible polynomial with integer coefficients, all of whose roots lie in the interval $I$. Then $\|P\|_{I}^{*} \geq a_{d}^{-1 / d}$ for every monic integer polynomial $P$, so that $t_{\mathrm{M}}(I) \geq a_{d}^{-1 / d}$. Furthermore, if $\|P\|_{I}^{*}=a_{d}^{-1 / d}$, then $t_{\mathrm{M}}(I)=a_{d}^{-1 / d}$ and $|P(\beta)|^{1 / \operatorname{deg} P}=a_{d}^{-1 / d}$ for every root $\beta$ of $Q$, and $\operatorname{Res}(P, Q)= \pm 1$. 
The proof follows straight from the classical fact that, for the conjugates $\beta_{i}$ of $\beta$,

$$
\operatorname{Res}(P, Q)=a_{d}^{\operatorname{deg} P} \prod_{i=1}^{d} P\left(\beta_{i}\right)
$$

is a nonzero integer, giving

$$
\|P\|_{I}^{*} \geq\left(\prod_{i}\left|P\left(\beta_{i}\right)\right|^{1 / \operatorname{deg} P}\right)^{\frac{1}{d}} \geq a_{d}^{-1 / d}|\operatorname{Res}(P, Q)|^{\frac{1}{d \operatorname{deg} P}} \geq a_{d}^{-1 / d} .
$$

This result is a variant of a similar one in the theory of $t_{\mathbb{Z}}(I)$; see Lemma 7.1

We call such a value $a_{d}^{-1 / d}$ in Lemma BPP an obstruction for $I$, with obstruction polynomial $Q(x)$. From Lemma BPP we see that $t_{\mathrm{M}}(I)$ is bounded below by the supremum of all such obstructions. If this supremum is attained by some value $a_{d}^{-1 / d}$ coming from $Q(x)=a_{d} x^{d}+\cdots+a_{0}$, then we say $a_{d}^{-1 / d}$ is a maximal obstruction, and $Q(x)$ is a maximal obstruction polynomial. It is not known whether such a polynomial exists for all intervals $I$ of length less than 4 (see Conjecture 2.3).

We say that the monic integer polynomial $P(x)$ is an optimal monic integer Chebyshev polynomial for $I$ if $\|P\|_{I}^{*}=t_{\mathrm{M}}(I)$. If $I$ has a maximal obstruction $a_{d}^{-1 / d}$ with $t_{\mathrm{M}}(I)=a_{d}^{-1 / d}$ and an optimal monic integer Chebyshev polynomial $P$, then we say that $P$ attains the maximal obstruction $a_{d}^{-1 / d}$.

Throughout this paper, $P(x)$ will denote a monic integer polynomial, $Q(x)$ a nonmonic integer polynomial, and $R(x)$ any integer polynomial.

We have seen that one very nice property of the monic integer transfinite diameter, not shared by its nonmonic cousin, is that often exact values can be computed for $t_{\mathrm{M}}(I)$. In all cases where this has been done, including Theorem 1.1, it was achieved by finding a maximal obstruction polynomial $Q$ and a corresponding optimal monic integer Chebyshev polynomial $P$. Simple examples of this are given ([2, Theorem 1.5]) by the intervals $I=[0,1 / n]$ for $n \geq 2$, where $Q(x)=n x-1$ is a maximal obstruction polynomial and $P(x)=x$ is an optimal monic integer Chebyshev polynomial. For $n=1, t_{\mathrm{M}}([0,1])=\frac{1}{2}$, with $Q(x)=2 x-1$ and $P(x)=x(x-1)$. This was the case too in [2, Section 5] in the proof of the Farey Interval Conjecture for small-denominator intervals.

A much less obvious example is the interval $I=[-0.3319,0.7412]$, of length 1.0731. Here, we have $t_{\mathrm{M}}(I)=\|P\|_{I}^{*}=7^{-1 / 3} \approx 0.522$, with maximal obstruction polynomial $7 x^{3}-7 x^{2}+1$ and where $P$ is the optimal monic integer Chebyshev polynomial

$$
\begin{aligned}
P(x)= & x^{276507}(x-1)^{29858}\left(x^{2}+x-1\right)^{14929} \\
& \left(x^{5}-17 x^{4}+24 x^{3}-8 x^{2}-2 x+1\right)^{28848} \\
& \left(x^{7}-117 x^{6}+194 x^{5}-70 x^{4}-31 x^{3}+18 x^{2}+x-1\right)^{7935} \\
& \left(x^{8}-4 x^{7}+97 x^{6}-172 x^{5}+78 x^{4}+20 x^{3}-18 x^{2}+1\right)^{9795} \\
& \left(x^{8}-34 x^{7}+164 x^{6}-208 x^{5}+65 x^{4}+33 x^{3}-18 x^{2}-x+1\right)^{5846} \\
& \left(x^{8}-7 x^{7}+2 x^{6}-x^{5}-10 x^{4}+28 x^{3}-15 x^{2}-2 x+2\right)^{1148}
\end{aligned}
$$

of degree 670320. (Tighter endpoints for this interval, and its length, can be computed by solving the equation $P(x)= \pm\left(7^{-1 / 3}\right)^{\operatorname{deg} P}$.) The discovery of this polynomial required the use of Lemma 6.1 below. 
For the nonmonic transfinite diameter $t_{\mathbb{Z}}$, Pritsker [13, Theorem 1.8] has recently proved that no integer polynomial $R(x)$ can attain $\|R(x)\|_{I}^{*}=t_{\mathbb{Z}}(I)$, this value possibly being achieved only by a normalized product of infinitely many polynomials. An immediate consequence of his result is the following.

Proposition 2.1. If an interval I has an optimal monic integer Chebyshev polynomial, then $t_{\mathrm{M}}(I)>t_{\mathbb{Z}}(I)$.

A fundamental question for both the monic and nonmonic integer transfinite diameter of an interval is whether its value can be computed exactly. In [2, Conjecture 5.1], Borwein et al. make a conjecture for Farey intervals (intervals $\left[\frac{b_{1}}{c_{1}}, \frac{b_{2}}{c_{2}}\right]$ where $b_{1}, b_{2}, c_{1}, c_{2} \in \mathbb{Z}$ and $\left.b_{2} c_{1}-b_{1} c_{2}=1\right)$ concerning the exact value of their monic transfinite diameter.

Conjecture BPP (Farey interval conjecture [1, p. 82], 2, Conjecture 5.1]). Suppose that $\left[\frac{b_{1}}{c_{1}}, \frac{b_{2}}{c_{2}}\right]$ is a Farey interval, neither of whose endpoints is an integer. Then

$$
t_{\mathrm{M}}\left(\left[\frac{b_{1}}{c_{1}}, \frac{b_{2}}{c_{2}}\right]\right)=\frac{1}{\min \left(c_{1}, c_{2}\right)} .
$$

Borwein et al. verify their conjecture for all Farey intervals having the denominators $c_{1}, c_{2}$ less than 22 . In Section 8 we extend the verification to some infinite families of Farey intervals (Theorems 8.2 and 8.3 ).

We next investigate what happens to $t_{\mathrm{M}}([0, b])$ when $b$ is close to $\frac{1}{n}$. For these intervals, some surprising things happen. Using the polynomial $P(x)=x$, we know that $t_{\mathrm{M}}([0, b]) \leq b<\frac{1}{n}$ if $b<\frac{1}{n}$. In fact it appears likely that $t_{\mathrm{M}}([0, b])$, clearly a nondecreasing function of $b$, has a left discontinuity at $t=1 / n(n>1)$. On the other hand, we show in Theorem 9.1 that $t_{\mathrm{M}}$ is locally constant on an interval of positive length $\delta_{n}$ to the right of $\frac{1}{n}$. Further, Theorem 9.2 gives much larger values for $\delta_{n}$ for $n=2,3$ and 4 , as well as an upper bound for $\delta_{2}$.

In fact, more may be true.

Conjecture 2.1 (Zero-endpoint interval conjecture). If $I=[0, b]$ is an interval with $b \leq 1$, then $t_{\mathrm{M}}(I)=1 / n$, where $n=\max \left(2,\left\lceil\frac{1}{b}\right\rceil\right)$ is the smallest integer $n \geq 2$ for which $1 / n \leq b$.

What little we know about $t_{\mathrm{M}}([0, b])$ for $b>1$ is given in Theorem 9.2(c), (d).

Both Conjecture BPP and Conjecture 2.1 are a consequence of the following conjecture.

Conjecture 2.2 (Maximal obstruction implies $t_{\mathrm{M}}(I)$ conjecture). If an interval $I$ of length less than 4 has a maximal obstruction $m$, then $t_{\mathrm{M}}(I)=m$.

We were at first tempted to conjecture here that $t_{\mathrm{M}}(I)$, as well as equaling its maximal obstruction, is always attained by some monic integer polynomial. However, the following counterexample eliminates this possibility in general.

Counterexample 2.1. The polynomial $7 x^{3}+4 x^{2}-2 x-1$ is a maximal obstruction polynomial for the interval $I=[-0.684,0.517]$. However, there is no monic integer polynomial $P$ with $\|P\|_{I}^{*}$ equal to the maximal obstruction $7^{-1 / 3}$ for $I$.

This result is proved in Section 10.

Our next result proves the existence of maximal obstructions for many intervals. 
Theorem 2.1. Every interval not containing an integer in its interior has a maximal obstruction.

Based on Conjecture 2.2 and Theorem 2.1 we make the following conjecture.

Conjecture 2.3 (Maximal obstruction conjecture). Every interval of length less than 4 has a maximal obstruction.

We do not have much direct evidence for this conjecture. However, our next conjecture, Conjecture 2.4 implies it. To describe this implication, we need the following notion, taken from Flammang, Rhin and Smyth [5]. An irreducible polynomial $Q(x)=a_{d} x^{d}+\cdots+a_{0} \in \mathbb{Z}[x]$ with $a_{d}>0$, all of whose roots lie in an interval $I$, and for which $a_{d}^{-1 / d}$ is greater than the (nonmonic) transfinite diameter $t_{\mathbb{Z}}(I)$, is called a critical polynomial for $I$. Here we are interested only in nonmonic critical polynomials.

It may be that every interval of length less than 4 has infinitely many nonmonic critical polynomials; see Proposition 2.2 below. We make the following weaker conjecture.

Conjecture 2.4 (Critical polynomial conjecture). Every interval of length less than 4 has at least one nonmonic critical polynomial.

From Theorem 2.1, this conjecture is true for intervals not containing an integer. For intervals $I$ of length less than 4 that do contain an integer (say 0 ), then, since $t_{\mathbb{Z}}(I)<1$, the polynomial $x$ is a critical polynomial for $I$. Thus 'nonmonic' is an important word in this conjecture.

In Theorem 7.1 we prove that Conjecture 2.4 implies Conjecture 2.3. $\quad$ More interestingly, we also prove in Corollary 7.1 that Conjecture 2.2 and Conjecture 2.3 together imply Conjecture 2.4 .

We observe in passing the following conditional result for the integer transfinite diameter $t_{\mathbb{Z}}$.

Proposition 2.2. Suppose that an interval I has infinitely many critical polynomials $Q_{i}(x)=a_{d_{i}, i} x^{d_{i}}+\cdots+a_{0, i}$. Then

$$
t_{\mathbb{Z}}(I)=\inf _{i} a_{d_{i}, i}^{-\frac{1}{d_{i}}}
$$

This result is proved in Section 7, Montgomery [11, p. 182] conjectured this result unconditionally for the interval $I=[0,1]$.

\section{UPPER AND LOWER BOUNDS FOR $L_{-}(t)$ AND $L_{+}(t)$ FOR FIXED $t$}

The following lemma contains some simple properties, as well as alternative definitions, of $L_{-}$and $L_{+}$.

Lemma 3.1. We have

(a) $L_{-}(t) \leq L_{+}(t)$ for $t \geq 0$;

(b) $L_{-}(t)=0$ for $0 \leq t \leq \frac{1}{2}$;

(c) $L_{+}(t) \geq 2 t$ for $0 \leq t \leq 1 / 2$;

(d) $L_{-}(t)=\sup _{I}\left\{d: t_{\mathrm{M}}(I) \leq t\right.$ for all $I$ with $\left.|I|=d\right\}$ for $t \geq \frac{1}{2}$;

(e) $L_{+}(t)=\inf _{I}\left\{d: t_{\mathrm{M}}(I)>t\right.$ for all $I$ with $\left.|I|=d\right\}$ for $t \geq 0$;

(f) $L_{+}(t)=L_{-}(t)=4 t$ for $t \geq 1$. 
Proof. First note that, by [2, equation (1.11)], $t_{\mathrm{M}}(I)=\frac{1}{2}$ for the zero-length interval $\left[\frac{1}{2}, \frac{1}{2}\right]$, from which (b) follows.

Part (c) follows from the fact that $\|x\|_{[-t, t]}^{*}=t$.

To prove (d), take $t \geq \frac{1}{2}$. Then the set

$$
S:=\left\{d: t_{\mathrm{M}}(I) \leq t \text { for all } I \text { with }|I|=d\right\}
$$

contains 0 (by (b)), so is nonempty. Put $s=\sup _{d} S$, and take $d \in S$. Since $I^{\prime} \subset I$ implies that $t_{\mathrm{M}}\left(I^{\prime}\right) \leq t_{\mathrm{M}}(I)$ ([2, Prop. 1.3]), any $d^{\prime}$ with $0 \leq d^{\prime}<d$ also lies in $S$, so that $S=[0, s)$ or $[0, s]$. Hence $L_{-}(t) \geq s$. On the other hand, for each $d>s$ there is an interval $I$ with $|I|=d$ and $t_{\mathrm{M}}(I)>t$. Hence $L_{-}(t) \leq d$, giving $L_{-}(t)=s$.

Now (a) follows straight from (b) and (d). The proof of (e), similar to that of (d), is left as an exercise for the reader.

Finally, part (f) follows from the fact that for $|I| \geq 4$ we have $t_{\mathrm{M}}(I)=t_{\mathbb{Z}}(I)=$ $\operatorname{cap}(I)=\frac{|I|}{4}($ see for instance [2] $)$.

Next, we give a simple lemma, needed for applying Proposition 3.1 below.

Lemma 3.2. Suppose that $I_{i}=\left[a_{i}, b_{i}\right] \quad(i=1, \ldots, n)$ are intervals with $a_{1}<a_{2}<$ $\cdots<a_{n}=a_{1}+1$, and put $M:=\max _{i=1}^{n-1}\left(b_{i+1}-a_{i}\right), m:=\min _{i=1}^{n-1}\left(b_{i}-a_{i+1}\right)$. Then

(a) Any interval of length at least $M$ contains an integer translate of some $I_{i}$.

(b) Any interval of length at most $m$ is contained in an integer translate of some $I_{i}$.

Proof. Given an interval $I$ of length $\ell$, we can, after translation by an integer, assume that $I=[a, b]$, where $a_{j} \leq a<a_{j+1}$, for some $j<n$.

(a) Suppose that $\ell \geq M$. Then $b_{j+1} \leq a_{j}+M \leq a+\ell$, so that $\left[a_{j+1}, b_{j+1}\right] \subset$ $[a, a+\ell]$.

(b) Suppose that $\ell \leq m$. Then $b_{j} \geq a_{j+1}+m>a+\ell$, so that $[a, a+\ell] \subset\left[a_{j}, b_{j}\right]$.

The following proposition will be used to obtain explicit upper and lower bounds for $L_{-}(t)$ and $L_{+}(t)$ for particular values of $t$.

\section{Proposition 3.1.}

(a) If $Q(x)=a_{d} x^{d}+\cdots+a_{0}$, with integer coefficients and $a_{d}>1$, has roots spanning an interval of length $\ell$, then for any $t<a_{d}^{-1 / d}$ we have

$$
L_{-}(t) \leq \ell \text {. }
$$

(b) Suppose that we have a finite set of polynomials $Q_{i}(x)=a_{d_{i}, i} x^{d_{i}}+\cdots+a_{0, i}$ with all $a_{d_{i}, i}^{-1 / d_{i}}>t$ with the property that every interval of length $\ell$ contains an integer translate of the roots of at least one of the polynomials $Q_{i}$. Then

$$
L_{+}(t) \leq \ell
$$

(c) Suppose that we have a finite set of intervals $I_{i}$ such that for each $I_{i}$ there is a monic integer polynomial $P_{i}$ with $\left\|P_{i}\right\|_{I_{i}}^{*} \leq t$. Suppose too that every interval of length $\ell$ is contained in an integer translate of some $I_{i}$. Then

$$
L_{-}(t) \geq \ell \text {. }
$$


(d) If $\|P\|_{I}^{*}=t$ for some monic integer polynomial $P$ and interval $I$ of length $\ell$, then

$$
L_{+}(t) \geq \ell
$$

Proof.

(a) Given such a $Q(x), \ell$ and interval $I$ of length $\ell$, and $t<a_{d}^{-1 / d}$, then from Lemma BPP we have $t_{\mathrm{M}}(I) \geq a_{d}^{-1 / d}>t$ so that, from the definition of $L_{-}(t)$, we have $L_{-}(t) \leq \ell$.

(b) Suppose that every interval $I$ of length $\ell$ contains some integer translate of the set of roots of some $Q_{i}$. Then, by Lemma BPP, $t_{\mathrm{M}}(I) \geq a_{d_{i}, i}^{-1 / d_{i}}>t$. Hence $t_{\mathrm{M}}\left(I^{\prime}\right)>t$ for any interval of length $\left|I^{\prime}\right| \geq \ell$, and so $L_{+}(t) \leq \ell$.

(c) Here, for every interval $I$ of length $\ell$ with $I+r \subset I_{i}$, say (with $r \in \mathbb{Z}$ ), we have

$$
t>\left\|P_{i}\right\|_{I_{i}}^{*} \geq\left\|P_{i}\right\|_{I+r}^{*}=\left\|P_{i}(x+r)\right\|_{I}^{*} \geq t_{\mathrm{M}}(I),
$$

so that any $I^{\prime}$ with $t_{\mathrm{M}}\left(I^{\prime}\right)>t$ has $\left|I^{\prime}\right|>\ell$. Hence $L_{-}(t) \geq \ell$.

(d) If $\|P\|_{I}^{*}=t$ and $|I|=\ell$, then $t_{\mathrm{M}}(I) \leq t$, so that $L_{+}(t) \geq \bar{\ell}$.

TABLE 1. Obstruction polynomials used for Theorem 1.2 to prove that $L_{+}\left(\frac{1}{2}\right)<1.4715$.

\begin{tabular}{|r|l|l|}
\hline$i$ & Polynomials $Q_{i}$ & Intervals $\left[a_{i}, b_{i}\right]$ \\
\hline 1 & $7 x^{3}+7 x^{2}-1$ & {$[-0.737,0.328]$} \\
2 & $57 x^{6}+81 x^{5}+6 x^{4}-32 x^{3}-9 x^{2}+3 x+1$ & {$[-0.728,0.494]$} \\
3 & $7 x^{3}+4 x^{2}-2 x-1$ & {$[-0.684,0.517]$} \\
4 & $59 x^{6}+28 x^{5}-43 x^{4}-15 x^{3}+11 x^{2}+2 x-1$ & {$[-0.669,0.528]$} \\
5 & $3 x^{2}-1$ & {$[-0.577,0.577]$} \\
6 & $59 x^{6}-28 x^{5}-43 x^{4}+15 x^{3}+11 x^{2}-2 x-1$ & {$[-0.528,0.669]$} \\
7 & $7 x^{3}-4 x^{2}-2 x+1$ & {$[-0.517,0.684]$} \\
8 & $57 x^{6}-81 x^{5}+6 x^{4}+32 x^{3}-9 x^{2}-3 x+1$ & {$[-0.494,0.728]$} \\
9 & $7 x^{3}-7 x^{2}+1$ & {$[-0.328,0.737]$} \\
10 & $63 x^{6}-136 x^{5}+72 x^{4}+16 x^{3}-17 x^{2}+1$ & {$[-0.310,1.115]$} \\
11 & $63 x^{6}-146 x^{5}+91 x^{4}+7 x^{3}-18 x^{2}+x+1$ & {$[-0.285,1.141]$} \\
12 & $58 x^{6}-139 x^{5}+90 x^{4}+6 x^{3}-18 x^{2}+x+1$ & {$[-0.285,1.178]$} \\
13 & $59 x^{6}-147 x^{5}+105 x^{4}-3 x^{3}-18 x^{2}+2 x+1$ & {$[-0.271,1.184]$} \\
14 & $63 x^{6}-159 x^{5}+115 x^{4}-4 x^{3}-19 x^{2}+2 x+1$ & {$[-0.260,1.197]$} \\
15 & $15 x^{4}-29 x^{3}+13 x^{2}+x-1$ & {$[-0.244,1.208]$} \\
16 & $57 x^{6}-171 x^{5}+153 x^{4}-21 x^{3}-21 x^{2}+3 x+1$ & {$[-0.228,1.228]$} \\
17 & $15 x^{4}-31 x^{3}+16 x^{2}-1$ & {$[-0.208,1.244]$} \\
18 & $63 x^{6}-219 x^{5}+265 x^{4}-126 x^{3}+14 x^{2}+5 x-1$ & {$[-0.197,1.260]$} \\
19 & $59 x^{6}-207 x^{5}+255 x^{4}-127 x^{3}+18 x^{2}+4 x-1$ & {$[-0.184,1.271]$} \\
20 & $58 x^{6}-209 x^{5}+265 x^{4}-136 x^{3}+20 x^{2}+4 x-1$ & {$[-0.178,1.285]$} \\
21 & $63 x^{6}-232 x^{5}+306 x^{4}-171 x^{3}+34 x^{2}+2 x-1$ & {$[-0.141,1.285]$} \\
22 & $63 x^{6}-242 x^{5}+337 x^{4}-204 x^{3}+48 x^{2}-1$ & {$[-0.115,1.310]$} \\
\hline
\end{tabular}


TABLe 2. Optimal monic integer Chebyshev polynomials used for Theorem 1.2 to prove that $L_{-}\left(\frac{1}{2}\right) \geq 1.008848$.

\begin{tabular}{|c|c|c|}
\hline$i$ & Polynomials $P_{i}$ & Intervals $I_{i}$ \\
\hline 1 & $\begin{array}{l}x^{1600}\left(x^{3}-4 x^{2}+1\right)^{36}\left(x^{4}+4 x^{3}-4 x^{2}-x+1\right)^{55} \\
\left(x^{8}+236 x^{7}-96 x^{6}-167 x^{5}+64 x^{4}+39 x^{3}-14 x^{2}-3 x+1\right)^{39} \\
\left(x^{8}+372 x^{7}-196 x^{6}-249 x^{5}+129 x^{4}+55 x^{3}-28 x^{2}-4 x+2\right)^{20}\end{array}$ & {$[-0.5142,0.5613]$} \\
\hline 2 & $\begin{array}{l}x^{2121}\left(x^{3}-4 x^{2}+1\right)^{77}\left(x^{4}-10 x^{3}+5 x^{2}+2 x-1\right)^{84} \\
\left(x^{7}-43 x^{6}-11 x^{5}+44 x^{4}+2 x^{3}-12 x^{2}+1\right)^{160}\end{array}$ & {$[-0.4501,0.5783]$} \\
\hline 3 & $\begin{array}{l}x^{12446}\left(x^{2}+x-1\right)^{199}\left(x^{4}-7 x^{3}+5 x^{2}+x-1\right)^{909} \\
\left(x^{6}-53 x^{5}+46 x^{4}+10 x^{3}-14 x^{2}+1\right)^{640}\end{array}$ & {$[-0.4388,0.5912]$} \\
\hline 4 & $\begin{array}{l}x^{312924}\left(x^{4}-7 x^{3}+5 x^{2}+x-1\right)^{45312} \\
\left(x^{4}+8 x^{3}-8 x^{2}+1\right)^{217}\left(x^{4}+9 x^{3}-7 x^{2}-x+1\right)^{23800}\end{array}$ & {$[-0.4267,0.6401]$} \\
\hline 5 & $\begin{array}{l}x^{17556}\left(x^{5}+16 x^{4}-22 x^{3}+5 x^{2}+3 x-1\right)^{2256} \\
\left(x^{4}+8 x^{3}-8 x^{2}+1\right)^{899}\end{array}$ & {$[-0.3797,0.6847]$} \\
\hline 6 & $\begin{array}{l}x^{49329424964}(x-1)^{6557517120}\left(x^{2}+x-1\right)^{70328} \\
\left(x^{4}+8 x^{3}-8 x^{2}+1\right)^{4916965515} \\
\left(x^{5}-17 x^{4}+24 x^{3}-8 x^{2}-2 x+1\right)^{5952478752} \\
\left(x^{5}+16 x^{4}-22 x^{3}+5 x^{2}+3 x-1\right)^{541825536}\end{array}$ & {$[-0.3241,0.7100]$} \\
\hline 7 & $\begin{array}{l}x^{114080}(x-1)^{9324}\left(x^{4}+8 x^{3}-8 x^{2}+1\right)^{529} \\
\left(x^{4}+9 x^{3}-9 x^{2}+1\right)^{2852} \\
\left(x^{8}+172 x^{7}-440 x^{6}+377 x^{5}-82 x^{4}-47 x^{3}+21 x^{2}+x-1\right)^{8184} \\
\left(x^{8}+214 x^{7}-531 x^{6}+440 x^{5}-90 x^{4}-54 x^{3}+23 x^{2}+x-1\right)^{6072}\end{array}$ & {$[-0.3064,0.7344]$} \\
\hline 8 & $\begin{array}{l}x^{15200}(x-1)^{5192}\left(x^{4}+9 x^{3}-9 x^{2}+1\right)^{192} \\
\left(x^{8}+172 x^{7}-440 x^{6}+377 x^{5}-82 x^{4}-47 x^{3}+21 x^{2}+x-1\right)^{1587}\end{array}$ & {$[-0.2943,0.7401]$} \\
\hline 9 & $\begin{array}{l}x^{3136}(x-1)^{1768}\left(x^{6}+3 x^{5}+6 x^{4}-18 x^{3}+9 x^{2}+x-1\right)^{32} \\
\left(x^{8}+172 x^{7}-440 x^{6}+377 x^{5}-82 x^{4}-47 x^{3}+21 x^{2}+x-1\right)^{91}\end{array}$ & {$[-0.2752,0.7645]$} \\
\hline 10 & $\begin{array}{l}x^{146704}(x-1)^{85868}\left(x^{2}+x-1\right)^{6369} \\
\left(x^{6}+3 x^{5}+6 x^{4}-18 x^{3}+9 x^{2}+x-1\right)^{1768}\end{array}$ & {$[-0.2622,1.1030]$} \\
\hline
\end{tabular}

Proof of Theorem 1.2, Applying Proposition 3.1(a) with $Q(x)=7 x^{3}-7 x^{2}+1$, we have

$$
L_{-}\left(\frac{1}{2}\right) \leq \ell=1.064961507 .
$$

Here, a more precise value could be determined by calculating the span of the roots of $Q(x)$ to a higher precision.

We apply Proposition 3.1(b) and Lemma 3.2(a) using the polynomials $Q_{i}$ of Table 1. with the intervals $\left[a_{i}, b_{i}\right]$ containing their roots. (Here, the endpoints listed in Table 1 are approximations of the minimal and maximal root of the obstruction polynomial in question. A higher precision was used for the computation of the upper bound of $L_{+}\left(\frac{1}{2}\right)<1.4715$.) We put $Q_{23}(x)=Q_{1}(x-1)$, whose roots are contained in $\left[a_{23}, b_{23}\right]:=\left[a_{1}+1, b_{1}+1\right]$, and apply the proposition to the 23 polynomials $Q_{1}, \ldots, Q_{23}$. Each has $a_{d}^{-1 / d}>\frac{1}{2}$. Then because $\max _{i=1}^{22}\left(b_{i+1}-a_{i}\right)=$ $b_{16}-a_{15}=1.4715$, any interval $I$ of length $|I|>1.4715$ must, by Lemma 3.2(a), contain an integer translate of some interval $\left[a_{i}, b_{i}\right]$, and so all the roots of the corresponding polynomial $Q_{i}$. Hence $L_{+}\left(\frac{1}{2}\right)<1.4715$. 
We apply Proposition 3.1(c) by starting with the 10 intervals $I_{i}(i=1, \ldots, 10)$ in Table 2, and putting $I_{i}=1-I_{21-i}$ and $P_{i}(x)=P_{21-i}(1-x)$ for $i=11, \ldots, 20$, with $I_{21}=1+I_{1}$ and $P_{21}(x)=P_{1}(x-1)$. (Here again, the endpoints listed in Table 2 are approximations only. To find more accurate values, we would solve for the roots of $P(x)= \pm\left(\frac{1}{2}\right)^{\operatorname{deg} P}$. Higher precision values were used to compute the lower bound $L_{-}\left(\frac{1}{2}\right)>1.008848$.) Each polynomial $P_{i}$ listed has a critical point at $\frac{1}{2}$ (and also at $-\frac{1}{2}$ in the case of the last polynomial), with $P_{i}\left(\frac{1}{2}\right)= \pm\left(\frac{1}{2}\right)^{\operatorname{deg} P_{i}}$. The value of $\left|P_{i}(x)\right|$ at all other critical points, as well as at the interval endpoints, is strictly less than $\left(\frac{1}{2}\right)^{\operatorname{deg} P_{i}}$. This shows in each case that $\left\|P_{i}\right\|_{I_{i}}^{*}=\frac{1}{2}$. Then all 21 intervals $I_{i}$ have $t_{\mathrm{M}}\left(I_{i}\right)=\frac{1}{2}$ and, writing $I_{i}=\left[a_{i}, b_{i}\right](i=1, \ldots, 21)$, we have

$$
\min _{i=1}^{20}\left(b_{i}-a_{i+1}\right)=b_{5}-a_{6}>1.008848 .
$$

From this it follows by Lemma 3.2(b) that every interval $I$ of length less than 1.008848 is a subinterval of an integer translate of some $I_{i}$, so that $t_{\mathrm{M}}(I) \leq\left\|P_{i}\right\|_{I}^{*} \leq$ $\left\|P_{i}\right\|_{I_{i}}^{*}=\frac{1}{2}$. This proves part (a) of the theorem.

Part (b) of the theorem follows by applying Proposition [3.1(d) with $P(x)=$ $x^{2}-x$. We then have, for $I=\left[\frac{1-\sqrt{2}}{2}, \frac{1+\sqrt{2}}{2}\right]$, that $\|P\|_{I}^{*}=\frac{1}{2}$, so that $L_{+}(t) \geq|I|=$ $\sqrt{2}$.

\section{General Bounds For $L_{-}(t)$ And $L_{+}(t)$}

In this section we find upper and lower bounds for $L_{-}(t)$ and $L_{+}(t)$, valid for $t$ from 0.5 to close to 0.9 . Our first result gives the upper bounds.

\section{Theorem 4.1.}

(a) For all $t_{i}$ and $\ell_{i}^{-}$in Table 3 and for all $t<t_{i}$ we have $L_{-}(t)<\ell_{i}^{-}$.

(b) For all $t_{i}$ and $\ell_{i}^{+}$in Table 4 and for all $t<t_{i}$ we have $L_{+}(t)<\ell_{i}^{+}$.

TABLE 3. Upper bounds for $L_{-}(t)$. Here $L_{-}(t)<\ell_{i}^{-}$for $t<t_{i}$, where $\ell_{i}^{-}$is the span of the roots of the $i$ th polynomial (see Theorem 4.1).

\begin{tabular}{|l|l|l|l|}
\hline$i$ & Polynomial $Q_{i}$ & $t_{i}$ & $\ell_{i}^{-}$ \\
\hline 1 & $7 x^{3}+7 x^{2}-1$ & $\frac{1}{\sqrt[3]{7}} \approx 0.522$ & 1.064961507 \\
2 & $3 x^{2}-1$ & $\frac{1}{\sqrt[2]{3}} \approx 0.577$ & 1.154700538 \\
3 & $5 x^{3}+3 x^{2}-2 x-1$ & $\frac{1}{\sqrt[3]{5}} \approx 0.584$ & 1.390656045 \\
4 & $2 x^{2}-1$ & $\frac{1}{\sqrt[2]{2}} \approx 0.707$ & 1.414213562 \\
5 & $3 x^{4}-2 x^{3}-4 x^{2}+x+1$ & $\frac{1}{\sqrt[4]{3}} \approx 0.759$ & 2.173182852 \\
6 & $2 x^{3}-4 x^{2}+1$ & $\frac{1}{\sqrt[3]{2}} \approx 0.793$ & 2.306243643 \\
7 & $2 x^{4}-8 x^{3}+8 x^{2}-1$ & $\frac{1}{\sqrt[4]{2}} \approx 0.840$ & 2.613125930 \\
8 & $2 x^{5}-15 x^{4}+39 x^{3}-40 x^{2}+12 x+1$ & $\frac{1}{\sqrt[5]{2}} \approx 0.870$ & 2.982466529 \\
9 & $2 x^{6}-12 x^{5}+22 x^{4}-8 x^{3}-10 x^{2}+4 x+1$ & $\frac{1}{\sqrt[6]{2}} \approx 0.890$ & 3.131521012 \\
\hline
\end{tabular}


TABLE 4. Upper bounds for $L_{+}(t)$. Here $L_{+}(t)<\ell_{i}^{+}$for $t<t_{i}$, where $\ell_{i}^{+}$is the span of the roots of the $i$ th polynomial (see Theorem 4.1).

\begin{tabular}{|r|c|c||c|c|c|}
\hline$i$ & $t_{i}$ & $\ell_{i}^{+}$ & $i$ & $t_{i}$ & $\ell_{i}^{+}$ \\
\hline 1 & $\frac{1}{\sqrt[6]{63}} \approx .501$ & 1.47149 & 31 & $\frac{1}{\sqrt[5]{15}} \approx .582$ & 1.71707 \\
2 & $\frac{1}{\sqrt[6]{60}} \approx .505$ & 1.47887 & 32 & $\frac{1}{\sqrt[3]{5}} \approx .585$ & 1.72578 \\
3 & $\frac{1}{\sqrt[5]{30}} \approx .506$ & 1.48183 & 33 & $\frac{1}{\sqrt[6]{24}} \approx .589$ & 1.78511 \\
4 & $\frac{1}{\sqrt[6]{59}} \approx .507$ & 1.48424 & 34 & $\frac{1}{\sqrt[5]{14}} \approx .590$ & 1.79006 \\
5 & $\frac{1}{\sqrt[4]{15}} \approx .508$ & 1.48823 & 35 & $\frac{1}{\sqrt[6]{23}} \approx .593$ & 1.80103 \\
6 & $\frac{1}{\sqrt[6]{58}} \approx .508$ & 1.49541 & 36 & $\frac{1}{\sqrt[4]{8}} \approx .595$ & 1.80333 \\
7 & $\frac{1}{\sqrt[6]{57}} \approx .510$ & 1.49802 & 37 & $\frac{1}{\sqrt[6]{22}} \approx .597$ & 1.80514 \\
8 & $\frac{1}{\sqrt[6]{56}} \approx .511$ & 1.50442 & 38 & $\frac{1}{\sqrt[6]{19}} \approx .612$ & 1.82308 \\
9 & $\frac{1}{\sqrt[4]{14}} \approx .517$ & 1.50918 & 39 & $\frac{1}{\sqrt[4]{7}} \approx .615$ & 1.82808 \\
10 & $\frac{1}{\sqrt[6]{51}} \approx .519$ & 1.51232 & 40 & $\frac{1}{\sqrt[6]{18}} \approx .618$ & 1.85414 \\
11 & $\frac{1}{\sqrt[3]{7}} \approx .523$ & 1.51409 & 41 & $\frac{1}{\sqrt[5]{11}} \approx .619$ & 1.86446 \\
12 & $\frac{1}{\sqrt[6]{48}} \approx .525$ & 1.54721 & 42 & $\frac{1}{\sqrt[6]{17}} \approx .624$ & 1.86909 \\
13 & $\frac{1}{\sqrt[5]{25}} \approx .525$ & 1.54825 & 43 & $\frac{1}{\sqrt[3]{4}} \approx .630$ & 1.87806 \\
14 & $\frac{1}{\sqrt[4]{13}} \approx .527$ & 1.55329 & 44 & $\frac{1}{\sqrt[6]{15}} \approx .637$ & 1.92375 \\
15 & $\frac{1}{\sqrt[6]{46}} \approx .528$ & 1.56522 & 45 & $\frac{1}{\sqrt[5]{9}} \approx .644$ & 1.92862 \\
16 & $\frac{1}{\sqrt[6]{45}} \approx .530$ & 1.57021 & 46 & $\frac{1}{\sqrt[4]{5}} \approx .669$ & 1.95815 \\
17 & $\frac{1}{\sqrt[5]{23}} \approx .534$ & 1.57066 & 47 & $\frac{1}{\sqrt[6]{11}} \approx .671$ & 2.03528 \\
18 & $\frac{1}{\sqrt[4]{12}} \approx .537$ & 1.57390 & 48 & $\frac{1}{\sqrt[3]{3}} \approx .693$ & 2.05072 \\
19 & $\frac{1}{\sqrt[5]{21}} \approx .544$ & 1.58148 & 49 & $\frac{1}{\sqrt{2}} \approx .707$ & 2.07313 \\
20 & $\frac{1}{\sqrt[4]{11}} \approx .549$ & 1.59285 & 50 & $\frac{1}{\sqrt[6]{7}} \approx .723$ & 2.46521 \\
21 & $\frac{1}{\sqrt[5]{20}} \approx .549$ & 1.60583 & 51 & $\frac{1}{\sqrt[5]{5}} \approx .725$ & 2.49418 \\
22 & $\frac{1}{\sqrt[6]{36}} \approx .550$ & 1.62320 & 52 & $\frac{1}{\sqrt[6]{6}} \approx .742$ & 2.55291 \\
23 & $\frac{1}{\sqrt[6]{34}} \approx .556$ & 1.63662 & 53 & $\frac{1}{\sqrt[5]{4}} \approx .758$ & 2.58796 \\
24 & $\frac{1}{\sqrt[6]{33}} \approx .558$ & 1.64392 & 54 & $\frac{1}{\sqrt[4]{3}} \approx .760$ & 2.60202 \\
25 & $\frac{1}{\sqrt[5]{18}} \approx .561$ & 1.65596 & 55 & $\frac{1}{\sqrt[6]{5}} \approx .765$ & 2.61238 \\
26 & $\frac{1}{\sqrt[6]{32}} \approx .561$ & 1.65815 & 56 & $\frac{1}{\sqrt[6]{4}} \approx .794$ & 2.70928 \\
27 & $\frac{1}{\sqrt[4]{10}} \approx .562$ & 1.66032 & 57 & $\frac{1}{\sqrt[5]{3}} \approx .803$ & 2.89569 \\
28 & $\frac{1}{\sqrt[6]{31}} \approx .564$ & 1.66308 & 58 & $\frac{1}{\sqrt[6]{3}} \approx .833$ & 2.97756 \\
29 & $\frac{1}{\sqrt[5]{16}} \approx .574$ & 1.67218 & 59 & $\frac{1}{\sqrt[4]{2}} \approx .841$ & 2.98928 \\
30 & $\frac{1}{\sqrt{3}} \approx .577$ & 1.68244 & 60 & $\frac{1}{\sqrt[5]{2}} \approx .871$ & 3.23520 \\
\hline
\end{tabular}

The theorem is proved by applying Proposition 3.1(a) and (b) for a range of values in $[0.5,1]$. Here again, the diameter given in Table 3 can be computed more exactly by considering the difference between the maximal and minimal roots of the obstruction polynomial. For Table 4, a calculation similar to that done for Table 1 was done for each $t_{i}$. The rounding procedure was that used for Table 1, Then the monotonicity of $L_{-}(t)$ and $L_{+}(t)$ gives the result for all $t$ in this range.

For the lower bounds, we first define the normalized polynomial $P_{\alpha}$,

$$
P_{\alpha}(x)=(x(1-x))^{\frac{1-\alpha}{2}}\left(x^{2}-x-1\right)^{\frac{\alpha}{2}},
$$

of degree 1 , and let $\alpha^{*} \approx 0.4358$ be the root in $(0,1)$ of the equation

$$
4 \alpha^{\alpha}(1-\alpha)^{1-\alpha}=5^{\alpha} \text {. }
$$




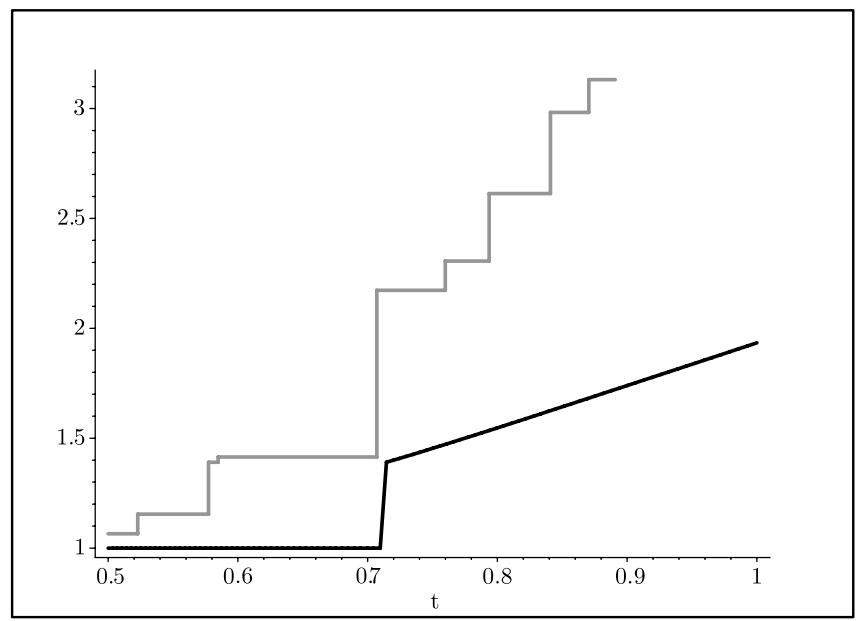

Figure 1. Upper and lower bounds for $L_{-}(t)$ (Theorem 4.1 and Proposition 4.1). Grey line, upper bound; black line, lower bound.

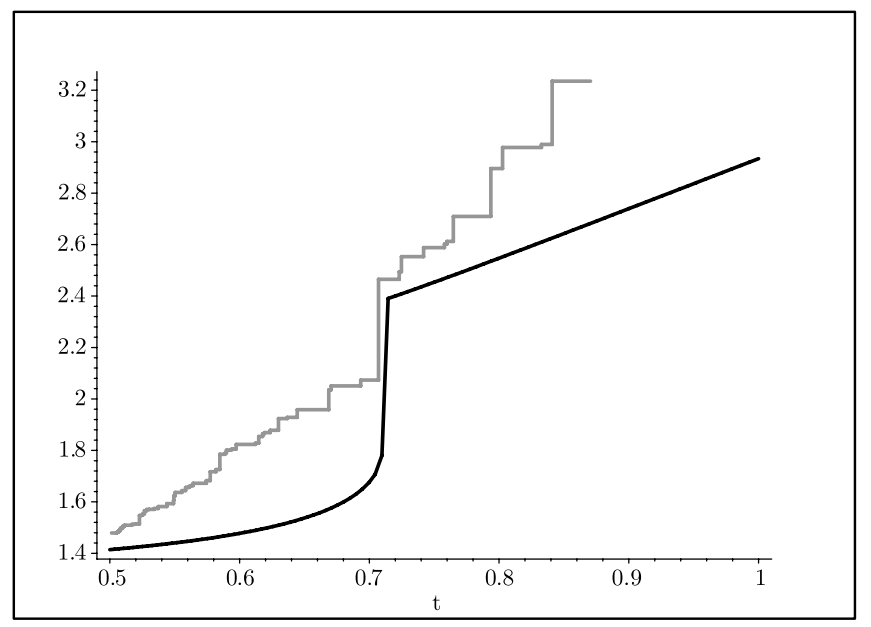

Figure 2. Upper and lower bounds for $L_{+}(t)$ (Theorem 4.1 and Proposition 4.11). Grey line, upper bound; black line, lower bound.

The following result gives the lower bounds.

Proposition 4.1. For $0 \leq \alpha \leq \frac{\ln 4}{\ln 5}$ we have

(a) $L_{+}\left(\frac{5^{\alpha / 2}}{2}\right) \geq \ell_{\alpha}$, where $\ell_{\alpha}$ is the root of $P_{\alpha}\left(\frac{1}{2}+\ell_{\alpha} / 2\right)=\frac{5^{\alpha / 2}}{2}$ in

$$
\begin{cases}(\sqrt{5}, \infty) & \text { if } \alpha>\alpha^{*}, \\ (1, \sqrt{5}) & \text { if } \alpha \leq \alpha^{*} .\end{cases}
$$

(b) $L_{-}\left(\frac{5^{\alpha / 2}}{2}\right) \geq \max \left(\ell_{\alpha}-1,1.008848\right)$.

For the proof, we need the following simple observation. 
Lemma 4.1. If $L_{+}(t) \geq \ell+1$, then $L_{-}(t) \geq \ell$.

This follows straight from the fact that, given an interval $I$ of length $\ell+1$, every interval of length $\ell$ has an integer translate that is a subinterval of $I$.

Proof of Proposition 4.1. It should first be pointed out that this proposition is in fact true for all $\alpha$, and not just those in the range specified. That being said, for $\alpha>\frac{\ln 4}{\ln 5}$ we would have $\frac{5^{\alpha / 2}}{2}>1$, in which case we could appeal to Lemma 3.1(f) for the exact answer.

(a) We will proceed to analyze $\left\|P_{\alpha}(x)\right\|_{I_{\ell}}^{*}$, picking $\alpha$ and $\ell$ such that, at the endpoints of the interval $I_{\ell},\left|P_{\alpha}(x)\right|$ equals the largest local maximum of $\left|P_{\alpha}(x)\right|$ in

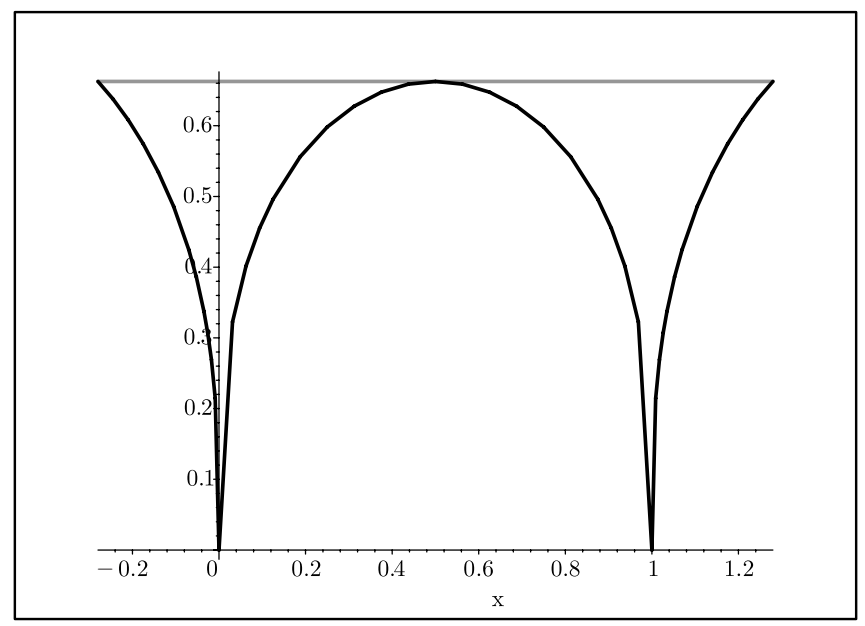

Figure 3. The normalized polynomial $P_{\alpha}(x)$ (see (4)) with $\alpha=0.35<\alpha^{*}, \ell_{\alpha} \approx 1.559$, and $t_{\mathrm{M}}\left(I_{\ell_{\alpha}}\right) \leq\left\|P_{\alpha}(x)\right\|_{I_{\ell_{\alpha}}}^{*} \approx 0.663$.

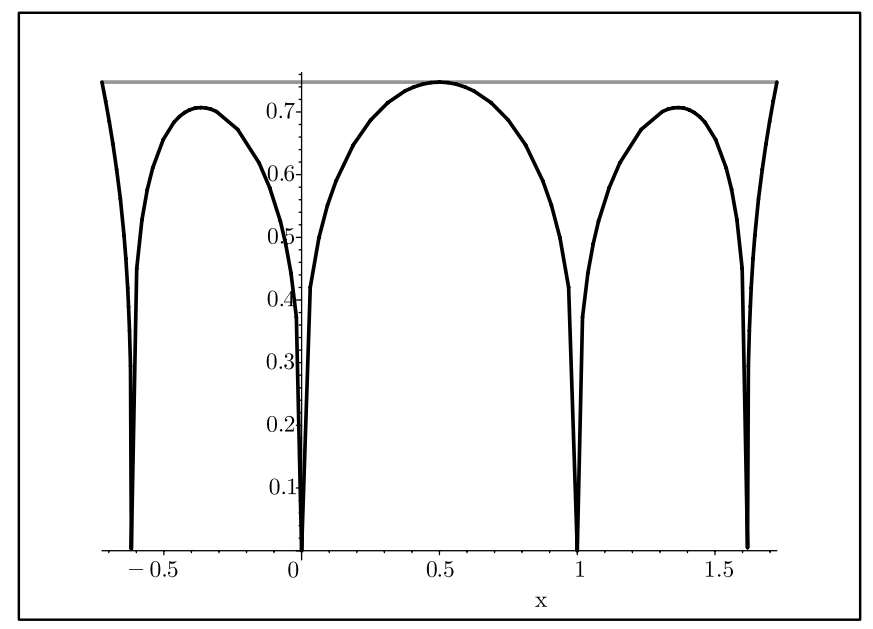

Figure 4. The normalized polynomial $P_{\alpha}(x)$ (see (4) with $\alpha=0.5>\alpha^{*}, \ell_{\alpha} \approx 2.449$, and $t_{\mathrm{M}}\left(I_{\ell_{\alpha}}\right) \leq\left\|P_{\alpha}(x)\right\|_{I_{\ell_{\alpha}}}^{*} \approx 0.748$. 
the interior of $I_{\ell}$. (Note that $P_{\alpha}$ is already normalized, so $\left\|P_{\alpha}\right\|_{I_{\ell}}^{*}=\left\|P_{\alpha}\right\|_{I_{\ell}}$.) (See Figures 3 and 4 .)

Note first that

$$
\left|P_{\alpha}\left(\frac{1}{2}+x / 2\right)\right|=\frac{5^{\frac{\alpha}{2}}}{2}\left|1-x^{2}\right|^{\frac{1-\alpha}{2}}\left|1-\frac{x^{2}}{5}\right|^{\frac{\alpha}{2}},
$$

which has a local maximum of $\frac{5^{\alpha / 2}}{2}$ at $x=0$, and a local maximum of $m_{\alpha}=$ $|1-\alpha|^{(-\alpha) / 2}|\alpha|^{\alpha / 2}$ at $x^{2}=5-4 \alpha$. Now the equation $m_{\alpha}=\frac{5^{\alpha / 2}}{2}$ has a root $\alpha$ defined by (5), with $m_{\alpha}>\frac{5^{\alpha / 2}}{2}$ for $\alpha<\alpha^{*}$ and $m_{\alpha}<\frac{5^{\alpha / 2}}{2}$ for $\alpha>\alpha^{*}$. Hence if $\alpha \geq \alpha^{*}$, then $\left|P_{\alpha}\left(\frac{1}{2}+x / 2\right)\right| \leq \frac{5^{\alpha / 2}}{2}$ for $x \leq \sqrt{5}$, so that $\left\|P_{\alpha}\right\|_{I_{\alpha}}^{*}=\frac{5^{\alpha / 2}}{2}$, where $I_{\alpha}=\left[\frac{1}{2}-\ell_{\alpha} / 2, \frac{1}{2}+\ell_{\alpha} / 2\right]$ with $\ell_{\alpha}$ the $\operatorname{root} \ell_{\alpha}>\sqrt{5}$ of $P_{\alpha}\left(\frac{1}{2}+\ell_{\alpha} / 2\right)=\frac{5^{\alpha / 2}}{2}$. However, if $\alpha<\alpha^{*}$, then we have the same result, but only for $\ell_{\alpha}$ the root in $(1, \sqrt{5})$ of $P_{\alpha}\left(\frac{1}{2}+\ell_{\alpha} / 2\right)=\frac{5^{\alpha / 2}}{2}$. This gives the lower bound $L_{+}\left(\frac{5^{\alpha / 2}}{2}\right) \geq \ell_{\alpha}$, but with a left discontinuity in $\ell_{\alpha}$ (as a function of $\alpha$ ) at $\alpha=\alpha^{*}$. A plot of this lower bound, along with the upper bounds from Theorem 4.1 and Table 4 , is given in Figure 2,

(b) We know that $L_{-}$is a nondecreasing function, and that $L_{-}\left(\frac{1}{2}\right) \geq 1.008848$. Combining these facts with Lemma 4.1 we get that

$$
L_{-}\left(\frac{5^{\alpha / 2}}{2}\right) \geq \max \left(\ell_{\alpha}-1,1.008848\right) .
$$

This is displayed numerically, along with the upper bounds from Theorem 4.1 and Table 3, in Figure 1.

\section{Intervals of length 1: Proof of Theorem 1.1}

Proof of Theorem 1.1. From Theorem 1.2 (a) we know that every interval $I$ of length $\ell \leq 1.008848$ has $t_{\mathrm{M}}(I) \leq \frac{1}{2}$. Now since every interval of length $\ell \geq 1$ has some integer translate that contains $\frac{1}{2}$, we have

$$
\frac{1}{2}=t_{\mathrm{M}}\left(\left\{\frac{1}{2}\right\}\right) \leq t_{\mathrm{M}}(I)
$$

for all such intervals, so that $t_{\mathrm{M}}(I)=\frac{1}{2}$ for all $I$ with $1 \leq|I| \leq 1.008848$.

If $b>1.064961507$, then again from Theorem[1.2(a), with the polynomial $Q(x)=$ $7 x^{3}+7 x^{2}-1$, there is an interval $I$ of length $b$ with $t_{\mathrm{M}}(I)>\frac{1}{2}$.

To complete the proof, note that for $b<1$

$$
t_{\mathrm{M}}([-b / 2, b / 2])=\sqrt{t_{\mathrm{M}}\left(\left[0, b^{2} / 4\right]\right)},
$$

by applying [2, Prop 1.4 with the polynomial $\left.x^{2}\right]$, and then

$$
\sqrt{t_{\mathrm{M}}\left(\left[0, b^{2} / 4\right]\right)} \leq \sqrt{b^{2} / 4}<\frac{1}{2}
$$

using the polynomial $P(x)=x$ on $\left[0, b^{2} / 4\right]$.

\section{Computational methods}

6.1. Finding optimal monic integer Chebyshev polynomials $\boldsymbol{P}$. We now describe how the polynomials of Table 2 were found. These are optimal monic integer polynomials $P$ having $\|P\|_{I}^{*}=\frac{1}{2}$ on various intervals of length just greater than 1. For these intervals, the maximal obstruction polynomial is $Q(x)=2 x-1$, and 
the maximal obstruction is $m=\frac{1}{2}$. The method applies more generally, however, to any interval $I$ having a maximal obstruction polynomial $Q$, so we shall describe the method for this more general situation. We suppose that the maximal obstruction is $m=a_{d}^{-1 / d}$, where $Q(x)=a_{d} x^{d}+\cdots+a_{0}$, so that we seek a monic integer polynomial $P$ with $\|P\|_{I}^{*}=m$.

First, potential factors of $P$ of small degree $k$ were identified using LLL [2, 8, 9]. The basis used was $\left[1, x, \ldots, x^{k}\right]$, with the inner product

$$
\left\langle R_{1}, R_{2}\right\rangle=\int_{I} R_{1}(x) R_{2}(x) d x+b_{k} c_{k} .
$$

Here $R_{1}(x)=b_{k} x^{k}+\cdots+b_{0}$ and $R_{2}(x)=c_{k} x^{k}+\cdots+c_{0}$. The $b_{k} c_{k}$ component of the inner product was inserted to discourage nonmonic polynomials from appearing in the basis returned by LLL. Now, at least one element in the basis will contain an $x^{k}$ term and, because of the $b_{k} c_{k}$ penalty, such an element is almost always monic. (In fact always in the examples we computed.) So we obtained a monic polynomial of degree $d$ with small $L_{2}$ norm, which usually also had a small supremum norm. These monic polynomials with small $L_{2}$ norm are not necessarily irreducible. At this point we examined each of their irreducible factors $f_{i}$, again monic polynomials, and applied Lemma 6.1(a) below to eliminate some of them. We then used the method of Borwein and Erdélyi [3] to search for exponents $\alpha_{i} \in \mathbb{N}$ such that $P^{1 / \operatorname{deg} P}:=$ $\prod_{i} f_{i}^{\alpha_{i} / \operatorname{deg} f_{i}}$ has the desired property $\|P\|_{I}^{*}=m$. To do this, we needed to minimize $t$ subject to the constraint

$$
\sum_{i} \frac{\alpha_{i}}{\operatorname{deg} f_{i}} \log \left(\left|f_{i}(x)\right|\right) \leq t
$$

for all $x \in I$ with $\sum_{i} \alpha_{i}=1,0 \leq \alpha_{i}$. Some additional constraints on the $\alpha_{i}$ that we made use of are given by Lemma 6.1(b), (c). The main difference between our application and the original one is that here the polynomials $f_{i}$ are all monic. By choosing a large number of points $x \in I$ to discretize the problem, we get a system of linear equations, on which the Simplex method can be used to get a good estimate of $\min (t)[3$, 7, 16]. In practice, with a high enough precision and a large enough number of sample points, we obtain $\min (t)=m$ exactly, and the corresponding $\alpha_{i}$ then give the required $P$. We then check that $P$ is indeed an optimal monic integer Chebyshev polynomial for $I$ by checking algebraically that $|P|^{1 / \operatorname{deg} P}=m$ at all roots of the maximal obstruction polynomial $Q$, and furthermore that all other local maxima of $|P|$ in this interval are strictly smaller than $m$.

The following lemma, used to help construct these polynomials $P$, specifies extra properties that their factors $f_{i}$ and normalized exponents $\alpha_{i}$ must have.

Lemma 6.1. Let $I$ be an interval that has a maximal obstruction polynomial $Q(x)=a_{d} x^{d}+\cdots+a_{0}$. Suppose further that $P(x)$ attains the maximal obstruction, and that $P(x)^{1 / \operatorname{deg} P}=\prod_{i} f_{i}^{\alpha_{i} / \operatorname{deg} f_{i}}$, with $\sum_{i} \alpha_{i}=1$. Then

(a) The resultant $\operatorname{Res}\left(f_{i}, Q\right)$ is equal to \pm 1 for every factor $f_{i} \in \mathbb{Z}[x]$ of $P$.

(b) For every root $\beta$ of $Q$ we have

$$
\sum_{i} \frac{\alpha_{i}}{\operatorname{deg} f_{i}} \times \frac{f_{i}^{\prime}(\beta)}{f_{i}(\beta)}=0 .
$$

(c) Fix a root $\beta \in \mathbb{R}$ of $Q$, and put $\hat{f}_{i}=\left|f_{i}(\beta)\right|^{1 / \operatorname{deg} f_{i}} \in \mathbb{R}$. Let $\mathcal{F}$ be the multiplicative subgroup of $\mathbb{R}_{>0}$ generated by $a_{d}$, and the $\hat{f}_{i}$ with $b_{1}=a_{d}$ and 
$b_{2}, \ldots, b_{k}$ an independent generating set for $\mathcal{F}$, with say $\hat{f}_{i}^{1 / \operatorname{deg} f_{i}}=\prod_{j} b_{j}^{c_{j, i}}$ for some integers $c_{j, i}$. Then

$$
\sum_{i} c_{j, i} \alpha_{i}= \begin{cases}-1 / d & \text { if } j=1 \\ 0 & \text { if } j>1 .\end{cases}
$$

Proof. We have $\prod_{i} P\left(\beta_{i}\right)= \pm 1 / a_{d}^{\mathrm{deg} P}$, where the product is taken over the roots $\beta_{i}$ of $Q$, so that, from (1) $\operatorname{Res}(P, Q)= \pm 1$. Then (a) follows from the fact that the resultant of a product with $Q$ is the product of the resultants with $Q$.

Part (a) follows from the fact that all the roots $\beta$ of $Q$ must be critical points of $P(x)$. Further, since $P(x)$ attains the maximal obstruction, we have from Lemma BPP that for all such $\beta$ we have $|P(\beta)|^{1 / \operatorname{deg} P}=a_{d}^{-1 / d}$, giving part (c).

Note that Lemma 6.1 simplifies considerably when the maximal obstruction polynomial is linear, say $a_{1} x-a_{0}$. Then it says that $f_{i}\left(\frac{a_{0}}{a_{1}}\right)= \pm a_{1}^{-\operatorname{deg} f_{i}}$ and with $P^{\prime}\left(\frac{a_{0}}{a_{1}}\right)=0$.

The independent generating set $b_{1}, \ldots, b_{k}$ for $\mathcal{F}$ was found using the integer relation-finding program PSLQ, which we used to search for linear integer relations between $\log a_{d}$ and $\log \hat{f}_{i}$.

As we have seen, the method for finding an optimal monic integer Chebyshev polynomial $P$ depends on first finding the (in practice there was only one) maximal obstruction polynomial for the interval. We now describe how to do this.

6.2. Finding obstruction polynomials $\boldsymbol{Q}$. The obstruction polynomial $7 x^{3}-$ $7 x^{2}+1$, as well as those listed in Tables 1 and 3, were found using the technique of Robinson 15. (see also [10, 17]). In this method, the aim is to search for all degree $d$ polynomials $Q(x)=a_{d} x^{d}+\cdots+a_{0}$ having all their roots in an interval $I_{0}$, for fixed degree, and fixed lead coefficient, $a_{d}$, with $a_{d} \leq 2^{d}$. We describe below how $I_{0}$ is chosen. Robinson's method uses the fact that for $k=1,2, \ldots, d-1$ the span of the roots of the $k$ th derivative of $Q$ is contained in the span of the roots of the $(k-1)$ th derivative of $Q$. In particular, these derivatives have all their roots in $I_{0}$.

Starting with the $(d-1)$ st derivative of $Q$, we get a range of possible valid values for $a_{d-1}$. Then consider the $(d-2)$ nd derivative to find valid ranges for $a_{d-2}$. Continuing in this fashion, we obtain a list of polynomials, each one having all its roots in $I_{0}$. We now sieve this list, first by eliminating all polynomials that are reducible or that have integer content greater than 1. Having obtained a list of irreducible polynomials, we can then prune it further, as follows. If $Q(x)$ and $R(x)$ are both irreducible polynomials, with the same degree and lead coefficient, and the span of the roots of $R(x)$ contains the roots of $Q(x)$, then for any interval $I$ where $R(x)$ is an obstruction polynomial, $Q(x)$ is also an obstruction polynomial, and hence $R(x)$ is not needed.

After construction of these polynomials, we can, for fixed $d, a_{d}$, and $t<a_{d}^{-1 / d}$, find an upper bound for $L_{-}(t)$ by finding the polynomial $Q$ whose roots have the smallest span, and then appeal to Proposition 3.1(a). This was done in Table 3 , formalized in Theorem 4.1(a), and displayed in Figure 1.

Similarly, given this list of polynomials, we can compute the least $\ell$ such that any interval of length $\ell$ will contain an integer translate of at least one of the polynomials in our list. Then with Proposition 3.1(b) we get an upper bound for $L_{+}(t)$. For 
given $\ell$, we must choose $I_{0}$ carefully. If $I_{0}$ is too short, we might miss an important obstruction polynomial. On the other hand, if $I_{0}$ is long, we will find, along with the obstruction polynomials we seek, (possibly multiple) integer translates of these polynomials. This is inefficient, as we end up doing more calculations than we need to do. So we wish to pick $I_{0}$ so that it is long enough to ensure that we have all important obstruction polynomials, and yet small enough that we are not doing more work than necessary. We do this by ensuring that $I_{0}$, the interval which contains the roots of the polynomials we have found, has the property that $\left|I_{0}\right|$ is just greater than $\ell+1$. This ensures that there are no other useful obstruction polynomials that we might have missed, since any obstruction polynomial having a span of length $\ell$ will then have some integer translate lying in $I_{0}$. (We might have to rerun the calculation if $\left|I_{0}\right|$ is too small, based on the current value of $\ell$.) We can achieve tighter upper bounds for $L_{+}(t)$ by considering the list of all obstruction polynomials we found such that $a_{d}^{-1 / d} \geq t$.

This computation was done for $t=\frac{1}{2}$ (Table 1 and Theorem 1.2) and also for 20 other values of $t$ (Table 4, Theorem 4.1(b) and Figure 21). To save space, the list of relevant polynomials for each $t$ is not given in the table. (This information is available upon request from the authors.)

\section{Critical polynomials: Results and proofs}

We first establish a relationship between critical polynomials and maximal obstructions. We define a maximal nonmonic critical polynomial of an interval $I$ to be a critical polynomial $Q(x)=a_{d} x^{d}+\cdots+a_{0}$ such that the value $a_{d}^{-1 / d}$ is maximal for $Q$ within the set of nonmonic critical polynomials for $I$. Such a polynomial is well defined, as a result of the following theorem.

Theorem 7.1. Suppose that an interval I has a nonmonic critical polynomial. Then I has a maximal nonmonic critical polynomial, $Q(x)=a_{d} x^{d}+\cdots+a_{0}$ say, and furthermore $Q$ is also a maximal obstruction polynomial, so that $a_{d}^{-1 / d}$ is the maximal obstruction.

To prove this result, we will apply the following version of a classical lemma.

Lemma 7.1 ([1, p. 77]). Let $Q(x)$ and $R(x)$ be two (not necessarily monic) integer polynomials. Further suppose that $Q(x)=a_{d} x^{d}+\cdots+a_{0}$ is a critical polynomial for the interval $I$, and that $R(x)$ satisfies $\|R\|_{I}^{*}<a_{d}^{-1 / d}$. Then $Q$ divides $R$.

Proof. From (11) and (2), with $R(x)$ replacing $P(x)$, we must have $\operatorname{Res}(Q, R)=$ 0.

This result, essentially known to early workers on integer transfinite diameter (Gorškov, Sanov, Trigub, Aparicio Bernardo, and others), has appeared in the literature in various forms; see for instance Chudnovsky [4, Lemma 2.3], Montgomery 11. Chapter 10], Borwein and Erdélyi [3], and Flammang, Rhin and Smyth [5].

Proof of Theorem 7.1. We first observe that nonmonic critical polynomials are obstruction polynomials. Conversely, if an obstruction is greater than $t_{\mathbb{Z}}(I)$, then its associated polynomial is also a critical polynomial.

Assume that $I$ has a nonmonic critical polynomial, and consider the nonempty set $\mathcal{A}=\left\{a_{d}^{-1 / d}\right\}$ of obstructions coming from the nonmonic critical polynomials of $I$. Any integer polynomial $R(x)$ (not necessarily monic) must, by Lemma 7.1 , 
contain as factors all critical polynomials $Q$ whose obstructions $a_{d}^{-1 / d}$ are strictly greater than $\|R(x)\|_{I}^{*}$. Therefore $\|R(x)\|_{I}^{*} \geq \ell$ for any limit point $\ell$ of $\mathcal{A}$, and hence $t_{\mathbb{Z}}(I)=\ell$. So if $\mathcal{A}$ has a limit point, then it must be $\inf (\mathcal{A})$. $\operatorname{Thus} \sup (\mathcal{A})$ is attained, and there is a maximal nonmonic critical polynomial $Q$, say. Then $Q$ is also a maximal obstruction polynomial.

Corollary 7.1. Conjectures 2.3 and 2.2 together imply Conjecture 2.4 .

Proof. From the proof above, we see that an obstruction that is greater than $t_{\mathbb{Z}}(I)$ is associated to a critical polynomial. The existence of such an obstruction is a consequence of Conjectures 2.3 and 2.2 ,

Proof of Proposition 2.2. Now $t_{\mathbb{Z}}(I) \leq \inf _{i} a_{d_{i}, i}^{-1 / d_{i}}$, by the definition of a critical polynomial. But if this inequality were strict, then we could find an integer polynomial $R$ with $t_{\mathbb{Z}}(I) \leq\|R\|_{I}^{*}<\inf _{i} a_{d_{i}, i}^{-1 / d_{i}}$. But then, from Lemma 7.1, $R$ would have to be divisible by all the $Q_{i}$, which is impossible.

\section{Farey intervals and the proof of Theorem 2.1}

Every closed interval $I$ has a least positive integer $q$ such that some rational $p / q$ with $(p, q)=1$ lies in the interior of $I$. If $q \geq 2$, then $I$ belongs to a unique Farey interval $\left[\frac{b_{1}}{c_{1}}, \frac{b_{2}}{c_{2}}\right]$ whose endpoints are consecutive fractions in the Farey sequence of order $q-1$. We define this interval to be the minimal Farey interval containing I.

Theorem 2.1 follows directly from our next result.

Theorem 8.1. Let $I$ be an interval not containing an integer in its interior, and let $\left[\frac{b_{1}}{c_{1}}, \frac{b_{2}}{c_{2}}\right]$ be the minimal Farey interval containing I. Then $\left(c_{1}+c_{2}\right) x-\left(b_{1}+b_{2}\right)$ is a critical polynomial for $I$. Moreover, the maximal obstruction for $I$ is

$$
= \begin{cases}\frac{1}{c_{1}} & \text { if } c_{1} \geq 2, \frac{b_{1}}{c_{1}} \in I, \frac{b_{2}}{c_{2}} \notin I, \\ \frac{1}{c_{2}} & \text { if } c_{2} \geq 2, \frac{b_{1}}{c_{1}} \notin I, \frac{b_{2}}{c_{2}} \in I, \\ \frac{1}{\min \left(c_{1}, c_{2}\right)} & \text { if } c_{1} \geq 2, c_{2} \geq 2 \text { and } I=\left[\frac{b_{1}}{c_{1}}, \frac{b_{2}}{c_{2}}\right], \\ \frac{1}{c_{1}+c_{2}} & \text { otherwise. }\end{cases}
$$

Proof. Now the polynomial $Q(x)=\left(c_{1} x-b_{1}\right)^{c_{2}}\left(c_{2} x-b_{2}\right)^{c_{1}}$ has a local maximum of $\left(\frac{1}{c_{1}+c_{2}}\right)^{c_{1}+c_{2}}$ at $x=\frac{b_{1}+b_{2}}{c_{1}+c_{2}}$. Thus, by continuity, there exist integers $r_{1}$ and $r_{2}$ such that $R(x):=Q(x)^{r_{1}}\left(\left(c_{1}+c_{2}\right) x-\left(b_{1}+b_{2}\right)\right)^{r_{2}}$ has normalized supremum less than $\frac{1}{c_{1}+c_{2}}$. Hence $\left(c_{1}+c_{2}\right) x-\left(b_{1}+b_{2}\right)$ is an obstruction polynomial. Now $\frac{b_{1}+b_{2}}{c_{1}+c_{2}} \in I$, as otherwise $I$ would be contained in one of the Farey intervals $\left[\frac{b_{1}}{c_{1}}, \frac{b_{1}+b_{2}}{c_{1}+c_{2}}\right]$ or $\left[\frac{b_{1}+b_{2}}{c_{1}+c_{2}}, \frac{b_{2}}{c_{2}}\right]$.

Since the polynomials $\left(c_{1}+c_{2}\right) x-\left(b_{1}+b_{2}\right), c_{1} x-b_{1}$ and $c_{2} x-b_{2}$ are critical only if their roots are in $I$, and are, as factors of $R$, by Lemma 7.1 the only three possible maximal critical polynomials in this Farey interval, we get the final result.

Theorem 8.2. Let $\left[\frac{b_{1}}{c_{1}}, \frac{b_{2}}{c_{2}}\right]$ with $c_{1} \geq 2$ be a Farey interval, and suppose that $b_{1}^{2} \equiv \pm 1\left(\bmod c_{1}\right)$ and $b_{2}^{2} \equiv B\left(\bmod c_{2}\right)$, where $c_{1}^{2}|B|<c_{2}^{2}$. Then

$$
t_{\mathrm{M}}\left(\left[\frac{b_{1}}{c_{1}}, \frac{b_{2}}{c_{2}}\right]\right)=\frac{1}{c_{1}} .
$$


Proof. From [2, p. 1905] we have that there exists a monic quadratic integer polynomial $P(x)$ which has the property that $P\left(\frac{b_{1}}{c_{1}}\right)= \pm \frac{1}{c_{1}^{2}}$ and $P\left(\frac{b_{2}}{c_{2}}\right)=\frac{B}{c_{2}^{2}}$. Since its critical point is at a half integer, it is strictly monotonic on the Farey interval. Hence it attains its maximum at one of its endpoints, and $\left|P\left(\frac{b_{1}}{c_{1}}\right)\right|>\left|P\left(\frac{b_{2}}{c_{2}}\right)\right|$.

Theorem 8.3. Let $P(x)=x^{2}+a_{1} x+a_{0}$ be an irreducible integer polynomial with real roots. Then there exist infinitely many Farey intervals for which $P(x)$ attains the maximal obstruction.

Proof. We know (Pell's Equation) that the equation $x^{2}+a_{1} x y+a_{0} y^{2}= \pm 1$ has an infinite number of integer solutions $(x, y)=\left(b_{i}, c_{i}\right)$. These solutions have the property that $P\left(\frac{b_{i}}{c_{i}}\right)= \pm \frac{1}{c_{i}^{2}}$. Further, by choosing a suitable subsequence we may assume that both the $c_{i}$ and the $b_{i} / c_{i}$ are monotonically increasing. Thus for any interval $I:=\left[\frac{b_{i}}{c_{i}}, \frac{b_{i+1}}{c_{i+1}}\right]$ not containing a half-integer, we see that $P(x)$ attains the maximal obstruction $1 / c_{i}$ with $Q(x)=c_{i} x-b_{i}$, so that $t_{\mathrm{M}}(I)=1 / c_{i}$. This happens infinitely often as the $\frac{b_{i}}{c_{i}}$ tend to a root of $P(x)$.

We can find a $\frac{b}{c} \in\left[\frac{b_{i}}{c_{i}}, \frac{b_{i+1}}{c_{i+1}}\right]$ such that $\left[\frac{b_{i}}{c_{i}}, \frac{b}{c}\right]$ is a Farey interval, and hence $P(x)$ attains its maximal obstruction $1 / c_{i}$ on this interval.

It should be noted that this method of proof will not work for polynomials of degree 3 or higher, as the resulting Thue equation

$$
x^{n}+a_{n-1} x^{n-1} y+\cdots+a_{0} y^{n}= \pm 1
$$

has only a finite number of integer solutions [18].

\section{STUdY of $t_{\mathrm{M}}(b)$}

In this section we consider intervals $[0, b]$, with $t_{\mathrm{M}}(b)$ denoting $t_{\mathrm{M}}([0, b])$. Our first result for such intervals is a consequence of Theorem 8.1.

Corollary 9.1. Let $n \geq 2$ and $\frac{1}{n}<b<\frac{1}{n-1}$. Then $\frac{1}{n}$ is the maximal obstruction of $[0, b]$.

Theorem 9.1. For all $n \in \mathbb{N}$ there exists $\delta_{n}>\frac{2}{n+\sqrt{n^{2}-4}}-\frac{1}{n}$ such that for all $0 \leq \varepsilon \leq \delta_{n}$

$$
t_{\mathrm{M}}\left(\left[0, \frac{1}{n}+\varepsilon\right]\right)=\frac{1}{n} .
$$

Proof. Consider the polynomial $P_{n}(x)=x^{n^{2}-2}\left(x^{2}-n x+1\right)$. It has the following properties:

- $P_{n}\left(\frac{1}{n}\right)=\left(\frac{1}{n}\right)^{n^{2}}$

- $P_{n}(x)$ has a local maximum (with respect to $x$ ) at $x=\frac{1}{n}$;

- $P_{n}(x)$ is strictly increasing (with respect to $x$ ) on $\left[0, \frac{1}{n}\right]$;

- $P_{n}(x)$ has a root $\beta_{n}=\frac{2}{n+\sqrt{n^{2}-4}}$ strictly greater than $\frac{1}{n}$;

- $P_{n}(x)$ is strictly decreasing on $\left[\frac{1}{n}, \beta_{n}\right]$.

Let $\alpha_{n}$ be the minimal root, strictly greater than $\beta_{n}$, of the equation $\left|P_{n}(x)\right|$ $=\frac{1}{n^{n^{2}}}$. Thus $P_{n}(x)$ demonstrates that $t_{\mathrm{M}}\left(\alpha_{n}\right)=\frac{1}{n}$, where $\alpha_{n}>\beta_{n}=$ $2 /\left(n+\sqrt{n^{2}-4}\right)>\frac{1}{n}$.

Theorem 9.2. We have that 
(a) $t_{\mathrm{M}}(b)=\frac{1}{4}$ for $b \in\left[\frac{1}{4}, 0.303\right]$;

(b) $t_{\mathrm{M}}(b)=\frac{1}{3}$ for $b \in\left[\frac{1}{3}, 0.465\right]$;

(c) $t_{\mathrm{M}}(b)=\frac{1}{2}$ for $b \in\left[\frac{1}{2}, 1.26\right]$;

(d) $t_{\mathrm{M}}(1.328)>\frac{1}{2}$.

Hence, in the notation of Theorem 9.1, $0.76 \leq \delta_{2}<0.828, \delta_{3}>0.132$ and $\delta_{4}>$ 0.053 .

Proof. The optimal monic polynomials needed for parts (a) and (b) are given in Table 5. In each case they attain the maximal obstruction $\frac{1}{4}$ and $\frac{1}{3}$, respectively. As before, a slightly larger interval can be computed exactly, by solving $P(x)=$ $\pm\left(\frac{1}{4}\right)^{\operatorname{deg} P}$ or $P(x)= \pm\left(\frac{1}{3}\right)^{\operatorname{deg} P}$, respectively. Part (c) follows from the first part of Table 2 using the map $x \mapsto 1-x$, with the same comments to the exact values as above. Part (d) is proved by using Lemma BPP and the obstruction polynomial $7 x^{3}-14 x^{2}+7 x-1$. Here 1.328 is an approximation to its largest root, rounded up to ensure that (d) holds.

The factors used for the construction of the polynomials in Table 5 were found using the techniques discussed in Section 6, making use of the constraints given by Lemma 6.1.

Bounds have been given on the exponents of certain factors for large integer Chebyshev polynomials used for estimating $t_{\mathbb{Z}}(I)$. For example, for the interval $I=[0,1]$, Pritsker $\left[12\right.$ shows that $(x(1-x))^{\gamma}$, where $0.2961 \leq \gamma \leq 0.3634$, must appear as a factor in any polynomial $R$ (normalized to have degree 1 ), for which $\|R\|_{I}^{*}$ is sufficiently close to $t_{\mathbb{Z}}(I)$.

Following [5], we now determine a lower bound for $\gamma(b)$ such that $x^{\gamma(b)}$ must divide any normalized monic integer polynomial $P$ such that $\|P\|_{[0, b]}^{*}$ approximates $t_{\mathrm{M}}(b)$ sufficiently closely.

Suppose that the function $m(b)$ is an upper bound for $t_{\mathrm{M}}(b)$. Then by Proposition 5.3 and Lemma 5.2 of [5] we have that $\gamma(b)$ is bounded below by the least positive root of

$$
\frac{(1+x)^{1+x}}{(1-x)^{1-x}(2 x)^{2 x} b^{x}}=\frac{1}{m(b)}
$$

So in particular, if $t_{\mathrm{M}}(b)=\frac{1}{\lceil 1 / b\rceil}$ for $b \in[0,1]$ as in Conjecture 2.1, then our lower bound for $\gamma(b)$ would have infinitely many discontinuities in this range (Figure 5 , black lines). However, we know, by using the polynomial $x$, that we have a provable,

TABLE 5. Optimal monic integer polynomials used for the proof of Theorem 9.2 .

$$
\begin{aligned}
\hline t_{\mathrm{M}}(b)= & \frac{1}{4} \text { for } b \in\left[\frac{1}{4}, 0.303\right] \text { by } P(x) \\
= & x^{640}\left(x^{5}+432 x^{4}-456 x^{3}+179 x^{2}-31 x+2\right)^{47} \\
& \left(x^{7}+8760 x^{6}-13342 x^{5}+8388 x^{4}-2784 x^{3}+514 x^{2}-50 x+2\right)^{35} \\
t_{\mathrm{M}}(b)= & \frac{1}{3} \text { for } b \in\left[\frac{1}{3}, 0.465\right] \text { by } P(x) \\
= & x^{1652706720}\left(x^{7}-1233 x^{6}+2406 x^{5}-1913 x^{4}+791 x^{3}-179 x^{2}+21 x-1\right)^{118037088} \\
& \left(x^{8}+4842 x^{7}-10935 x^{6}+10355 x^{5}-5317 x^{4}+1594 x^{3}-278 x^{2}+26 x-1\right)^{156479575} \\
& \left(x^{8}+14184 x^{7}-34944 x^{6}+36442 x^{5}-20832 x^{4}+7041 x^{3}-1405 x^{2}+153 x-7\right)^{72166388} \\
& \left(x^{8}+7812 x^{7}-18072 x^{6}+17561 x^{5}-9271 x^{4}+2864 x^{3}-516 x^{2}+50 x-2\right)^{4378185} \\
&
\end{aligned}
$$




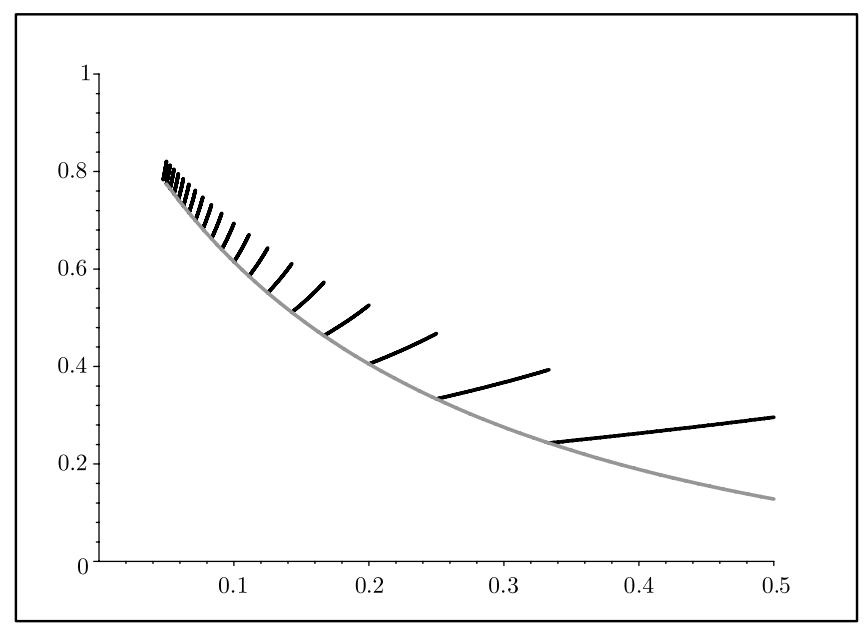

FiguRE 5. Lower bounds for $\gamma(b)$. Grey line-lower bound using $m(b)=b$ (known); solid line-lower bound assuming $m(b)=\frac{1}{\lceil 1 / b\rceil}$ (Conjecture 2.1).

albeit weaker, upper bound $m(b)=b$. This gives us a proven lower bound for $\gamma(b)$ (Figure 5, the grey line).

Proposition 9.1. We have $\lim _{b \rightarrow 0} \gamma(b)=1$.

Proof. Define

$$
T(x, b)=\frac{(1+x)^{1+x}}{(1-x)^{1-x}(2 x)^{2 x} b^{x}}-\frac{1}{b} .
$$

Now $T(x, b)$ has a positive local maximum at $x=\frac{1}{\sqrt{1+4 b}} \rightarrow 1$ as $b \rightarrow 0$, while $T(1-\sqrt{b}, b)<0$ for $0<b<0.04$, so that $T(x, b)=0$ has a root in $\left[1-\sqrt{b}, \frac{1}{\sqrt{1+4 b}}\right]$. Further, since $T(x, b)$ is increasing for $x \in\left[0, \frac{1}{\sqrt{1+4 b}}\right]$, this root is the least positive root of $T(x, b)=0$. Hence $\gamma(b)>1-\sqrt{b}$, giving the result.

\section{Proof of Counterexample 2.1}

For the proof of Counterexample 2.1 we need the following $p$-adic result.

Proposition 10.1. Suppose that $Q(x)=a_{d} x^{d}+\cdots+a_{0} \in \mathbb{Z}[x]$ is a maximal obstruction polynomial for the interval $I$, and that the maximal obstruction is attained by some monic integer polynomial $P(x)$. Then $\operatorname{gcd}\left(a_{0}, a_{d}\right)=1$ and, for every prime $p$ dividing $a_{d}$, we have

$$
\left|\frac{a_{d-i}}{a_{d}}\right|_{p} \leq\left|\frac{1}{a_{d}}\right|_{p}^{i / d} \quad(i=0, \ldots, d) .
$$

In particular, if $a_{d}$ is square-free, then $\frac{1}{a_{d}}(Q(x)-Q(0))$ has integer coefficients.

Here $|\cdot|_{p}$ is the usual $p$-adic valuation on $\mathbb{Q}$. For the proof, it is extended to $\overline{\mathbb{Q}}$.

Proof. Take $\beta$ to be any root of $Q(x)$, and $p$ any prime factor of $a_{d}$. Let $P(x)$ be of degree $m$. Then, as $P(x)$ attains the obstruction, $P(\beta)= \pm a_{d}^{-m / d}$, so that 
$|P(\beta)|_{p}=\left|1 / a_{d}\right|_{p}^{m / d}>1$. If $|\beta|_{p} \leq 1$, then $|P(\beta)|_{p} \leq 1$, a contradiction, as $P(x)$ has integer coefficients. Hence $|\beta|_{p}>1$ and $|P(\beta)|_{p}=|\beta|_{p}^{m}=\left|1 / a_{d}\right|_{p}^{m / d}$, giving

$$
|\beta|_{p}=\left|a_{d}\right|_{p}^{-1 / d} \text {. }
$$

Applying (6) for all roots $\beta_{j}$ of $Q(x)$ we get $\left|\prod_{j} \beta_{j}\right|_{p}=\left|1 / a_{d}\right|_{p}$. But also from $a_{d}^{-1} Q(x)=\prod_{j}\left(x-\beta_{j}\right)$ we have that $\left|\prod_{j} \beta_{j}\right|_{p}=\left|a_{0} / a_{d}\right|_{p}$. Hence $\left|a_{0}\right|_{p}=1$. Doing this for all $p \mid a_{d}$ we obtain $\left(a_{0}, a_{d}\right)=1$. Furthermore, if $\left|\frac{a_{d-i}}{a_{d}}\right|_{p}>\left|\frac{1}{a_{d}}\right|_{p}^{i / d}$ for any $i$, then the Newton polygon of $P$ (see for instance [19, p. 73]) tells us that $\left|\beta_{j}\right|_{p}>\left|1 / a_{d}\right|_{p}^{1 / d}$ for some $j$, contradicting (6).

In the case of $a_{d}$ square-free, $\left|\frac{1}{a_{d}}\right|_{p}^{i / d}<p$ for $1 \leq i<d$, so that $\left|\frac{a_{d-i}}{a_{d}}\right|_{p} \leq 1$, and hence, using all primes $p$ dividing $a_{d}$, we see that $\frac{a_{d-i}}{a_{d}}$ is an integer.

Proof of Counterexample 2.1. The fact that $7 x^{3}+4 x^{2}-2 x-1$ is a maximal obstruction polynomial for the interval $I=[-0.684,0.517]$ can be verified by showing that it is a critical polynomial. This follows from the fact that the polynomial

$$
\begin{aligned}
R(x)= & x^{28728}\left(5 x^{3}+4 x^{2}-x-1\right)^{3739}\left(7 x^{3}+4 x^{2}-2 x-1\right)^{1140} \\
& \left(x^{6}-24 x^{5}-20 x^{4}+10 x^{3}+9 x^{2}-x-1\right)^{420} \\
& \left(3 x^{5}+16 x^{4}+3 x^{3}-8 x^{2}-x+1\right)^{399}
\end{aligned}
$$

has $\|R\|_{I}^{*}<7^{-1 / 3}$, so that $t_{\mathbb{Z}}(I)<7^{-1 / 3}$. As $7 x^{3}+4 x^{2}-2 x-1$ has all its roots in $I$, it is therefore a critical polynomial. As always, the interval is an approximation only, and a tighter one can easily be computed.

We now claim that $7 x^{3}+4 x^{2}-2 x-1$ is the maximal nonmonic critical polynomial for $I$. For any critical polynomial $a_{d} x^{d}+\cdots+a_{0}$ for $I$ with $a_{d}^{-1 / d}>\|R\|_{I}^{*}$ must be a factor of $R$, by Lemma 7.1. But among the three nonmonic irreducible factors of $R, 7 x^{3}+4 x^{2}-2 x-1$ is the only one having all its roots within $I$, so it must indeed be the maximal nonmonic critical polynomial for $I$. By Theorem 7.1 this polynomial is the maximal obstruction polynomial. However, $\frac{1}{7}\left(7 x^{3}+4 x^{2}-2 x\right)$ does not have integer coefficients, so that, by Proposition 10.1, the interval has no optimal monic integer Chebyshev polynomial.

\section{Some final COMments on the COMputations AND Figures}

Consider Figure 1. We see that $L_{-}(t)=0$ for for $t<\frac{1}{2}$, and further that $L_{-}(t)=4 t$ for $t>1$. So in fact the area of interest is for $t$ between $\frac{1}{2}$ and 1. That being said, the upper bound is only given up to approximately 0.89 . This is because the upper bound from Proposition 3.1(a) is given by high degree polynomials with small lead coefficient. In our search, we compute only up to degree 6 . As $2^{-1 / 6} \approx 0.89$ this is the limit to our knowledge of the upper bound. If we wished to extend these calculations, we could extend the knowledge of the upper bound, but the computation time becomes excessive. For example, even if we computed up to degree 10, which is probably beyond our computational range, we would only get up to 0.933 . As it was, the computations up to degree 6 took over $3000 \mathrm{CPU}$ hours, and the computation time approximately triples for each additional degree. Similar comments apply to bounding $L_{+}(t)$ (Figure 2) for $t$ close to 1 . In this case, it actually turned out that none of the polynomials with lead 
coefficient 2 and degree 6 were useful in the calculations for such $t$, and hence we only get an upper bound for $L_{+}(t)$ for $t$ up to $t=2^{-1 / 5} \approx 0.871$.

While we know from Lemma 3.1 (c) that $L_{+}(t) \geq 2 t$ for $t \leq 1 / 2$, we do not know $L_{+}(t)$ exactly in this range. In order to get an upper bound for $L_{+}(t)$ in at least part of this range, it would in principle be possible to extend the calculation downwards from $t=\frac{1}{2}$. The lower bound of $\frac{1}{2}$ for $t$ was chosen, as we computed obstruction polynomials of degree $d$, with coefficients up to $2^{d}$. If we were to compute up to $3^{d}$ instead, we would be able to extend this graph down to $t=\frac{1}{3}$. This would, however, be a massive undertaking, because we would have $3^{6} / 2^{6}>11$ times as many possible lead coefficients. Furthermore, we observed that, for a given degree, the higher the lead coefficient was, the longer the computations took, so this factor 11 is probably an underestimate.

It may be possible to extend these calculations by somehow doing a more selective search for obstruction polynomials of higher degree or larger lead coefficients. This could lead to improved bounds

Lastly, consider Figure 5. This could very easily have been extended all the way to 0 . The reason that we chose not to do this is because the black lines of the hypothetical lower bound start to merge, and the figure becomes unreadable. (The lower bound jumps at every $\frac{1}{n}$, which get more frequent as $\frac{1}{n} \rightarrow 0$.)

\section{ACKNOWLEDGEMENT}

We thank the referee for helpful comments.

\section{REFERENCES}

[1] Peter Borwein. Computational excursions in analysis and number theory, Springer-Verlag, New York, 2002. MR1912495 (2003m:11045)

[2] P. B. Borwein, C. G. Pinner, and I. E. Pritsker, Monic integer Chebyshev problem, Math. Comp. 72 (2003), 1901-1916. MR.1986811 (2004e:11022)

[3] Peter Borwein and Tamás Erdélyi, The integer Chebyshev problem, Math. Comp. 65 (1996), no. 214, 661-681. MR.1333305 (96g:11077)

[4] G. V. Chudnovsky, Number theoretic applications of polynomials with rational coefficients defined by extremality conditions. Arithmetic and geometry, Vol. I, 61-105, Progr. Math., 35, Birkhäuser Boston, Boston, MA, 1983. MR0717590 (86c:11052)

[5] V. Flammang, G. Rhin, and C. J. Smyth, The integer transfinite diameter of intervals and totally real algebraic integers, J. Théor. Nombres Bordeaux 9 (1997), no. 1, 137-168. MR1469665 (98g:11119)

[6] G. M. Goluzin, Geometric theory of functions of a complex variable, Translations of Mathematical Monographs, Vol. 26, American Mathematical Society, Providence, R.I., 1969. MR0247039 (40:308)

[7] Laurent Habsieger and Bruno Salvy, On integer Chebyshev polynomials, Math. Comp. 66 (1997), no. 218, 763-770. MR.1401941 (97f:11053)

[8] Kevin G. Hare, Some applications of the LLL algorithm, Proceedings from the Maple Summer Workshop, 2002, Maple Software, Waterloo, 2002.

[9] A. K. Lenstra, H. W. Lenstra, Jr., and L. Lovász, Factoring polynomials with rational coefficients, Math. Ann. 261 (1982), no. 4, 515-534. MR0682664 (84a:12002)

[10] J.F. McKee and C.J. Smyth, Salem numbers of trace -2 and traces of totally positive algebraic integers, Proc. 6th. Algorithmic Number Theory Symposium (University of Vermont, 13-18 June 2004), Lecture Notes in Comput. Sci., vol. 3076, Springer, Berlin, 2004, pp. 327-337. MR2137365

[11] Hugh L. Montgomery, Ten lectures on the interface between analytic number theory and harmonic analysis. CBMS Regional Conference Series in Mathematics, 84. American Mathematical Society, Providence, RI, 1994. MR1297543(96i:11002) 
[12] Igor E. Pritsker, Chebyshev polynomials with integer coefficients, Analytic and geometric inequalities and applications, Math. Appl., vol. 478, Kluwer Acad. Publ., Dordrecht, 1999, pp. 335-348. MR.1785878 (2001h:30007)

[13] _ Small polynomials with integer coefficients, J. Anal. Math. 96 (2005), 151-190. MR2177184

[14] Thomas Ransford, Potential theory in the complex plane, London Mathematical Society Student Texts, vol. 28, Cambridge University Press, Cambridge, 1995. MR1334766 (96e:31001)

[15] Raphael M. Robinson, Algebraic equations with span less than 4, Math. Comp. 18 (1964), 547-559. MR0169374(29:6624)

[16] A. Schrijver, Theory of linear and integer programming, John Wiley \& Sons, Ltd., Chichester, 1986, A Wiley-Interscience Publication. MR0874114 (88m:90090)

[17] Christopher Smyth, Totally positive algebraic integers of small trace, Ann. Inst. Fourier (Grenoble) 34 (1984), no. 3, 1-28. MR0762691 (86f:11091)

[18] Vladimir G. Sprindžuk, Classical Diophantine equations, Lecture Notes in Mathematics, vol. 1559, Springer-Verlag, Berlin, 1993, Translated from the 1982 Russian original. MR 1288309 (95g:11017)

[19] Edwin Weiss, Algebraic number theory. McGraw-Hill Book Co., Inc., New York-San Francisco-Toronto-London, 1963 (Reprinted by Dover Publications, Inc., Mineola, NY, 1998.) MR:1635455 (99c:11129)

Department of Pure Mathematics, University of Waterloo, Waterloo, Ontario, CANADA N2L 3G1

E-mail address: kghare@math.uwaterloo.ca

School of Mathematics, University of Edinburgh, James Clerk Maxwell Building, King's Buildings, Mayfield Road, Edinburgh EH9 3JZ, United Kingdom

E-mail address: c.smyth@ed.ac.uk 